\title{
Brain Targeting of Duloxetine HCL via Intranasal Delivery of Loaded Cubosomal Gel: In vitro Characterization, ex vivo Permeation, and in vivo Biodistribution Studies
}

This article was published in the following Dove Press journal:

International Journal of Nanomedicine

Fatma Mohamed Elsenosy' Ghada Ahmed Abdelbary ${ }^{2}$ Ahmed Hassen Elshafeey (D) ${ }^{2}$ Ibrahim Elsayed (D) ${ }^{2,3}$

Ahmed Roshdy Fares ${ }^{2}$

'Department of Clinical Pharmacy, Children's Cancer Hospital, Cairo57357, Egypt; ${ }^{2}$ Department of Pharmaceutics and Industrial Pharmacy, Faculty of Pharmacy, Cairo University, Cairo, Egypt; ${ }^{3}$ Department of Pharmaceutical Sciences, College of Pharmacy and Thumbay Research Institute for Precision Medicine, Gulf Medical University, Ajman, United Arab Emirates
Correspondence: Ahmed Roshdy Fares Department of Pharmaceutics and Industrial Pharmacy, Faculty of Pharmacy, Cairo University, Kasr El Aini Street,

Cairo II562, Egypt

Tel +201288285866

Email ahmed.roshdy@pharma.cu.edu.eg
Purpose: Duloxetine (DLX) is dual serotonin and norepinephrine reuptake inhibitor suffering from limited bioavailability $(\approx 40 \%)$ due to extensive hepatic metabolism. This work aims to formulate and evaluate DLX intranasal thermoreversible cubosomal gels to enhance its bioavailability and ensure efficient brain targeting.

Materials and Methods: Cubo-gels were prepared by $3^{3}$ central composite design with three independent factors, lipid ratio (glycerol monooleate: glycerol tripalmitate), Pluronic F127\%, and Pluronic F68\%. The prepared formulations were evaluated for their particle size (PS), gelling temperature (GT), entrapment efficiency (EE\%), and in vitro release. The cubogel with the highest desirability $(0.88)$ was chosen as the optimized formulation. DLX cubogel was evaluated using differential scanning calorimetry, Fourier-transform infrared spectroscopy, X-ray powder diffraction, and transmission electron microscopy. Cytotoxicity study, ex vivo permeation study and in vivo bio-distribution study were conducted to evaluate the safety and efficacy of brain targeting.

Results: The optimum cubo-gel was composed of 3.76 lipid ratio, 20\% w/v PF127, and 5\% w/v PF68. It had PS of $265.13 \pm 9.85 \mathrm{~nm}$, GT of $32 \pm 0.05^{\circ} \mathrm{C}, \mathrm{EE} \%$ of $98.13 \pm 0.50 \%$, and showed controlled release behavior where 33\% DLX was released within 6 hrs. The plain in situ cubo-gel had a significantly higher $\mathrm{IC}_{50}$ compared to DLX solution and DLX-loaded in situ cubo-gel. The ex vivo permeation study showed 1.27 enhancement in the drug permeation from DLX in situ cubo-gel. According to the in vivo bio-distribution study in plasma and brain, the intranasal DLX in situ cubo-gel showed a 1.96 fold improvement in brain bioavailability compared to the intranasal solution. Its BTE\% and DTP\% were 137.77 and 10.5, respectively, indicating efficient brain targeting after intranasal administration.

Conclusion: Accordingly, intranasal DLX in situ cubo-gel can be considered as an innovative nano-carrier delivery system for bioavailability enhancement and efficient brain targeting of DLX to maximize its effect.

Keywords: duloxetine, central composite design, cubosomes, thermoreversible in situ gel, intranasal, brain targeting

\section{Introduction}

Duloxetine (DLX), \{(3S)-N-methyl-3-naphthalene-1-acyloxy-3-thiophene-2-ylpropan-1-amine $\}$, is a dual serotonin and norepinephrine reuptake inhibitor used in major depressive disorder. ${ }^{1}$ It is also used to manage urinary incontinence, fibromyalgia, and diabetic peripheral neuropathic pain. ${ }^{2}$ DLX is a biopharmaceutics 
classification system (BCS) class II drug, having limited aqueous solubility. DLX solubility was reported to be $2.68 \mathrm{mg} / \mathrm{mL}$ at $\mathrm{pH} 6.8^{3} \mathrm{DLX}$ is well absorbed after oral administration but shows a lag time of $2 \mathrm{~h}$ before starting the absorption and reaching its $t_{\max }$ after $6 \mathrm{~h}$ post-dosing. DLX has limited and variable bioavailability (40\% to $80 \%$ ) due to extensive hepatic metabolism, and it is also subjected to degradation in the acidic stomach medium, which leads to subtherapeutic levels. ${ }^{4,5}$

Intranasal administration is an advanced drug delivery route that allows effortless self-administration without sterile equipment. ${ }^{6}$ Fast drug delivery to the systemic circulation from the nasal cavity occurs due to drug absorption through a large single epithelial layer. ${ }^{7}$ The nasal route has privilege in brain targeting by direct drug transfer to the brain through neuronal and extracellular pathways and avoidance of the blood-brain barrier (BBB). ${ }^{8}$ The most significant advantage of the nasal route is improving drug bioavailability by bypassing firstpass hepatic metabolism, low enzymatic activity, and direct delivery to the systemic circulation. Also, the nasal delivery provides rapid onset of action, ease of administration, and non-invasiveness. On the other hand, the intranasal route suffers from limitations such as limited formulation volume that can be inserted into the nasal cavity, rapid mucociliary wash out which leads to short residence time, and poor absorption to hydrophilic and large molecules. These limitations can be overcome by the preparation of drug-loaded in situ cubosomal gel.

New nano-vesicular systems like liposomes, cubosomes, and niosomes are considered interesting candidates for nose to brain drug delivery. Their size and physical characteristics make them a new promising tool that can enhance drug residence time at the absorption site, protect the encapsulated drug from degradation, promote mucosal permeation, and control the release profile of the entrapped drug. 9

Cubosomes are one of the new nanocarriers. It consists of a bicontinuous lipid bilayer separating two networks of water channels. Cubosomes are composed of an amphiphilic polar lipid such as glycerol monooleate (GMO), which is the most common polar lipid used in cubosomes formulation in the presence of a stabilizer like Pluronic F127 (PF127). ${ }^{10}$ GMO can self-assemble in water to form a micellar structure when its concentration exceeds the critical micelle concentration (CMC). At higher concentrations, it forms a bicontinuous cubic structure. ${ }^{11,12}$ Cubosomes have many advantages like high entrapment efficiency, controlled drug release, biocompatibility, bioadhesive properties, and thermodynamic stability. ${ }^{13}$

In situ intranasal gel, which is a solution at room temperature $\left(<25^{\circ} \mathrm{C}\right)$ and forms a gel when inserted into the nasal cavity $\left(32^{\circ} \mathrm{C}\right.$ to $\left.34^{\circ} \mathrm{C}\right)$, prolongs the nasal residence time of the formulation so increases the penetration rate and improves the nasal absorption. ${ }^{14}$ In situ nasal gels are composed of thermoresponsive polymers like chitosan, pluronics, xyloglucans, and hydroxypropyl methylcellulose. ${ }^{15}$ Pluronic F127 (PF127) and Pluronic F68 (PF68) are the most frequent thermoresponsive polymers used. Pluronics are triblock copolymers soluble in water and composed of polyethylene oxide (PEO) and polypropylene (PPO) moieties. The ratio between PEO and PPO is responsible for hydrophobic and hydrophilic properties, respectively. ${ }^{12}$

Central composite design (CCD) is a practical statistical experimental design for studying the main effects of the experimental factors and their interactions using fewer experimental runs compared to a full factorial design. It covers many possible combinations and requires only three levels of each factor. CCD can be used to predict and optimize the responses to prepare the best formulation. $^{16,17}$

In a previous study, Alam et al examined the effect of the administration of an intranasal infusion containing DLX nanostructured lipid carriers (NLC) to improve the amount of DLX in the brain and plasma. ${ }^{18}$ In another study, Khatoon et al evaluated the intranasal administration of thiolated chitosan gel containing DLX proniosomes to improve its brain delivery. ${ }^{19}$

This study aims to formulate and characterize DLX in situ cubosomal gel to improve its bioavailability and enhance its brain targeting. A three-factor, three-level CCD was used to study the effects of different variables on the studied responses to prepare an optimized formulation. The independent variables selected were: Lipid ratio [Glycerol monooleate (GMO): Glycerol tripalmitate (GTP)] (A), PF127 percentage (B), and PF68 percentage (C). The dependent variables chosen in the study included: particle size (PS) $\left[\mathrm{Y}_{1}\right]$, gelling temperature (GT) $\left[\mathrm{Y}_{2}\right]$, entrapment efficiency percent (EE\%) $\left[\mathrm{Y}_{3}\right]$, and percent released after 6 hours $(\mathrm{Q} 6)\left[\mathrm{Y}_{4}\right]$. The optimum DLX in situ cubo-gel was physically characterized using transmission electron microscopy (TEM). Finally, the optimum formulation performance in brain targeting was characterized by conducting ex vivo permeation and in vivo bio-distribution studies. 
As per our knowledge, no previous studies prepared cubosomes containing GTP, combined with GMO, to investigate its effect on the $\mathrm{EE} \%$, Q6, and the brain targeting capability of the cubosomes placed in in situ gelling systems. This novel formulation aims to combine the advantages of the in situ gelling systems, namely, ease of intranasal instillation, high retention time, and sustained drug release after being converted into a gel; with the small particle size and lipophilicity of cubosomes to achieve better permeation and brain targeting of DLX.

\section{Materials and Methods Materials}

Duloxetine hydrochloride (DLX) was kindly supplied by EVA Pharma, Cairo, Egypt. Pluronic F127 (PF127), Pluronic F68 (PF68), Glycerol monooleate (GMO), and Glycerol tripalmitate (GTP) were purchased from SigmaAldrich, St. Louis, USA. All other chemicals and solvents were of analytical grade and used without further purification.

\section{Experimental Design}

A three-factor, three-level $\left(3^{3}\right) \mathrm{CCD}$ was conducted to statistically optimize the formulation variables of 15 formulations with 5 repetitions of the center point formulation (F8) per block using Design-Expert ${ }^{\circledR}$ software (version 7, Stat-Ease Inc., MN, USA). The independent variables were: Lipid ratio [ratio of GMO: GTP] (A), PF $127 \%$ (B), and PF68\% (C). The levels of the factors were selected as $(-1,0$, and +1$)$. The compositions of the formulations with their actual representative values are shown in Table 1. The dependant variables were particle size (PS) $\left[\mathrm{Y}_{1}\right]$, gelling temperature (GT) $\left[\mathrm{Y}_{2}\right]$, entrapment efficiency percent $(\mathrm{EE} \%)\left[\mathrm{Y}_{3}\right]$, and percent released after 6 hours (Q6) $\left[\mathrm{Y}_{4}\right]$. The polydispersity index (PDI) and zeta potential (ZP) were also measured for the prepared formulations. Desirability values were calculated based on the response surface analysis of the obtained data. They were used to select the optimized composition having as much as possible of the desired characteristics.

\section{Preparation of DLX in situ Cubo-Gels Preparation of Polymeric in situ Gelling Systems}

According to the design mentioned above, different Pluronic solutions were prepared according to the cold method previously described by Soga et al with some modifications. ${ }^{20}$ PF127 and PF68 were accurately weighed and solubilized in
Table I Composition of the $3^{3}$ CCD for DLX in situ Cubo-Gels

\begin{tabular}{|l|l|l|l|}
\hline \multirow{2}{*}{ Formula } & \multicolumn{3}{|l|}{ Factors Levels in Actual Values } \\
\cline { 2 - 4 } & $\begin{array}{l}\text { Lipid Ratio } \\
\text { (A) }\end{array}$ & $\begin{array}{l}\text { PFI 27 (\% w/v) } \\
\text { (B) }\end{array}$ & PF68 (\% w/v) \\
(C)
\end{tabular}

$10 \mathrm{~mL}$ distilled water by continuous stirring at $1000 \mathrm{rpm}$. The dispersion was left to hydrate overnight at $4^{\circ} \mathrm{C}$ to obtain a uniform, glassy solution. Table 1 shows the composition of different gelling systems prepared.

\section{Preparation of Thermosensitive Cubo-Gels}

Variable ratios of GMO and GTP were accurately weighed and mixed with $50 \mathrm{mg}$ of DLX. The lipid mixture was melt in a water bath at $70^{\circ} \mathrm{C}$ to obtain a clear lipid melt. The different ratios of GMO to GTP are shown in Table 1. The homogenous lipid melt is added drop wisely to the magnetically stirred Pluronic solution at $1000 \mathrm{rpm}$ and $25^{\circ} \mathrm{C}$ and left for 1 hour.

\section{Characterization of the Prepared in situ Cubo-Gels \\ PS, PDI, and ZP Analysis}

The dynamic light scattering technique was used to analyze the cubosomes PS (Zetasizer Nano ZS-90, Malvern Instruments, and Worcestershire, UK). Before measurement, $1 \mathrm{~mL}$ of each formulation was diluted with distilled water until being translucent. Additionally, PS distribution was assessed by measuring the PDI. Finally, physical stability was evaluated by analyzing the ZP of the diluted formulation samples. Triplicate measurements were 
provided for three isolated samples of each formulation; the average values were determined for each \pm standard deviation (SD).

\section{Measurement of Gelling Temperature (GT)}

GT was determined using the tilting method described by Zaki et al. ${ }^{21}$ An aliquot of $2 \mathrm{~mL}$ of the prepared formulation was transferred to a test tube and immersed in the water bath. The water bath temperature was increased gradually by one degree and allowed to equilibrate for 5 min at each new temperature. The sample was then examined for gelling. Gelling is confirmed when the meniscus of the sample is no longer moving when the test tube is tilted at a $90^{\circ}$ angle.

\section{Entrapment Efficiency Percent (EE\%)}

$\mathrm{EE} \%$ is a measurement of DLX content encapsulated in the cubosomes. EE\% was evaluated using the ultrafiltration method. An aliquot of $0.5 \mathrm{~mL}$ from each formulation, equivalent to $2.5 \mathrm{mg}$ DLX, was filtrated using a $0.22 \mu \mathrm{m}$ syringe filter (Nylon $25 \mathrm{~mm}$ Luer syringe filter). After filtration, the clear filtrate was diluted by distilled water, and the free DLX content was analyzed using a UV spectrophotometer at $\lambda_{\max } 286$. The EE\% was calculated using the following equation: ${ }^{22}$

$$
\text { Entrapment efficiency } \%=\left(\frac{C_{t}-C_{f}}{C_{t}}\right) \times 100
$$

where $\mathrm{C}_{\mathrm{t}}$ is the total DLX, and $\mathrm{C}_{\mathrm{f}}$ is the free DLX.

\section{DLX in vitro Release Profiles from the in situ Cubo-Gels}

In vitro release of DLX was evaluated using the membrane diffusion method. ${ }^{23}$ An aliquot of $1 \mathrm{~mL}$ of each formulation equivalent to $5 \mathrm{mg}$ DLX was transferred to a dialysis membrane hydrated overnight in the release medium (12-14 kDa, Sigma-Aldrich, St. Louis, USA). The dialysis membrane was then immersed in $45 \mathrm{~mL}$ phosphate buffer saline (PBS) $\mathrm{pH} 7.4$ (to maintain sink condition) and maintained at $37 \pm 0.5^{\circ} \mathrm{C}$. The medium was stirred at $50 \mathrm{rpm}$. At a defined time interval $(0.5,1,2,3,4,6 \mathrm{~h})$, $3 \mathrm{~mL}$ samples were withdrawn and immediately replaced by the same fresh medium volume. The withdrawn samples were analyzed by a UV spectrophotometer at $\lambda_{\max }$ $286 \mathrm{~nm}$.

\section{Optimized Formulation Selection}

The optimal formulation was selected using Design Expert $^{\circledR}$ software with the minimum PS, GT, and Q6 correlated with the maximum EE\%. Afterward, the optimized formulation was prepared and subjected to physical characterization using the same techniques to validate the results with the predetermined one. Finally, the optimized formulation was prepared and lyophilized for $24 \mathrm{~h}$ with a condenser temperature of $-45^{\circ} \mathrm{C}$ (Alpha 1-2 LD plus CHRIST, Germany) for more characterization.

\section{Characterization of the Optimized DLX in situ Cubo-Gel Formulation PS, PDI, ZP, GT, EE\% and in vitro DLX Release}

PS, PDI, ZP, GT, EE\%, and in vitro release of the optimized formulation were measured using the same methods and techniques previously used to characterize the initial set of formulations.

\section{Gelling Time}

The gelling time is the time required by the formulation for the transition from solution to gel when inserted into the nasal cavity. An aliquot of $1 \mathrm{~mL}$ formulation was transferred in a test tube then fitted horizontally in a water bath adjusted at $33 \pm 0.5^{\circ} \mathrm{C}$. The time required to form gel was recorded using a stopwatch. ${ }^{24}$

\section{$\mathrm{pH}$ Measurement}

An aliquot of $1 \mathrm{~mL}$ of the optimized formulation was diluted by $10 \mathrm{~mL}$ distilled water then the $\mathrm{pH}$ of the resulting solution was measured using a $\mathrm{pH}$ meter (Beckman Coulter, USA), which was previously calibrated using buffer $\mathrm{pH} 4$ then $\mathrm{pH} 7 .^{25}$

\section{Transmission Electron Microscope (TEM) Imaging}

The morphology, shape, and size of the optimum cubo-gel were photographed using TEM (JXA-840; JEOL, Tokyo, Japan). The cubo-gel was applied to the carbon-coated copper array, and then the HR-TEM detection of the cubosomes was conducted at an operating voltage of 200 $\mathrm{kV}$ in bright-field mode.

\section{Differential Scanning Calorimetry (DSC)}

DSC was performed on DLX loaded in situ cubo-gel and free DLX to detect any physical change in DLX after being entrapped in the cubosomes and to analyze its compatibility using (DSC-60, Shimadzu, Japan). Samples (3-4 mg) were placed in an aluminum pan and heated by a constant rate of $10^{\circ} \mathrm{C} / \mathrm{min}$, with an empty pan as a reference, in a nitrogen atmosphere to a temperature range $30^{\circ} \mathrm{C}-350^{\circ} \mathrm{C}$. 


\section{Fourier-Transform Infrared Spectroscopy (FTIR)}

DLX and drug-loaded in situ cubo-gel were analyzed by FTIR (Thermo Scientific Nicolet 6700, USA) to detect any possible interaction. The samples were compressed with $\mathrm{KBr}$ into circular disks. The samples were scanned in the range of 400 to $4000 \mathrm{~cm}^{-1}$.

\section{X-Ray Powder Diffraction (XRPD)}

$X$-ray diffraction patterns of free DLX and the optimized in situ cubo-gel were carried out using the X-ray diffractometer (model XD-610, Shimadzu, Kyoto, Japan) with $\mathrm{Cu}$ as tube node. The diffractograms were recorded under the following conditions: the voltage $45 \mathrm{kV}$, the current 30 $\mathrm{mA}$, the steps $0.02^{\circ}$, and the counting rate $0.5 \mathrm{~s} / \mathrm{step}$ at room temperature. Data were collected using scattering angle $(2 \theta)$ ranged from $4^{\circ}$ to $50^{\circ} .{ }^{10}$

\section{Cytotoxicity Assay}

The study was conducted on oral epithelial cells purchased from Nawah Scientific Inc., Cairo, Egypt. The cells were supported by Dulbecco's modified Eagle medium containing penicillin, streptomycin, and fetal bovine serum in concentrations $100 \mathrm{U} / \mathrm{mL}, 100 \mathrm{mg} / \mathrm{mL}$, and $10 \%$, respectively. ${ }^{26}$ Cell suspension ( $100 \mu \mathrm{L}$ aliquots) was incubated at $37^{\circ} \mathrm{C}$ under carbon dioxide for $24 \mathrm{~h}$ in a 96-well plate. Samples of DLX solution, the optimized plain in situ cubo-gel, and the optimized DLX loaded in situ cubo-gel, $100 \mu \mathrm{L}$ each, with different concentrations $(0.01,0.1,1$, $10,100 \mu \mathrm{g} / \mathrm{mL}$ ), were added to the cell suspensions. An aqueous solution of trichloroacetic acid $(150 \mu \mathrm{L}, 10 \% \mathrm{w} / \mathrm{v})$ was added to the cell after $72 \mathrm{~h}$ as a fixative agent. After $1 \mathrm{~h}$ of incubation at $4^{\circ} \mathrm{C}$, the cells were washed 5 times with distilled water, mixed with $70 \mu \mathrm{L}$ Sulforhodamine B solution $(0.4 \% \mathrm{w} / \mathrm{v})$, and incubated again at $25^{\circ} \mathrm{C}$ away from light for $10 \mathrm{~min}^{27}$ The cells were washed with $1 \% \mathrm{w} / \mathrm{v}$ acetic acid solution three times, left to dry overnight, and then mixed with TRIS buffer $(150 \mu \mathrm{L}, 10$ $\mathrm{mM})$. Finally, the absorbance was measured using a BMG LABTECH $^{\circledR}$ - FLUOstar Omega microplate reader (Ortenberg, Germany) at $540 \mathrm{~nm}$.

\section{Ex vivo DLX Permeation Study}

Ex vivo permeation study was used to determine the cubosomes ability to improve drug permeation through the nasal mucosa and effective brain targeting. The permeation of DLX from the optimized cubo-gel was compared to the DLX solution. The nasal membrane was obtained from Rahmani sheep nostrils with an average age of 6 months and a weight of $32 \mathrm{~kg} .^{22}$ The intact membrane was identified, separated, cleaned, and stored frozen. The excised membrane $(1 \mathrm{~cm}$ diameter $)$ was fixed at one end of the specially designed glass tube. The tube was fixed from the other end perpendicularly in a glass container containing $50 \mathrm{~mL}$ PBS (pH 7.4). An aliquot of $1 \mathrm{~mL}$ of the optimum formulation equivalent to $5 \mathrm{mg}$ DLX was added in the glass tube. The glass container was immersed in the horizontally shaking water bath at temperature $37^{\circ} \mathrm{C} \pm 0.5$ and $50 \mathrm{rpm}$ for $24 \mathrm{hr}$. A $3 \mathrm{~mL}$ sample was withdrawn at various time intervals $(0.5,1,2,3,4,6$, and $24 \mathrm{~h}$ ), and the volumes lost by the samples' withdrawal were replaced with fresh medium. The samples were analyzed by HPLC (Shimadzu, Tokyo, Japan). The mobile phase was composed of acetonitrile in $10 \mathrm{mM}$ phosphate buffer $\mathrm{pH} 4.5$ in the ratio of 55:45. The flow rate was $1 \mathrm{~mL} / \mathrm{min}$. DLX was detected through a UV detector (SPD-10 A, Shimadzu, Tokyo, Japan) at wavelength $288 \mathrm{~nm}^{5}$ All measurements were done in triplicate, and mean \pm SD was reported. The quantity of the drug permeated was calculated as a function of the surface area of the nasal membranes $\left(\mu \mathrm{g} / \mathrm{cm}^{2}\right)$. Then, the drug flux at $24 \mathrm{~h}\left(\mathrm{~J}_{24}\right)$ was calculated by this equation: $:^{22,28}$

$$
J_{24}=\frac{\text { Amount of drug permeated per unit area }}{\text { Time }}
$$

The drug permeated from the optimized formulation, and the drug solution was statistically compared by one-way ANOVA, then by Fisher's least significant difference test. The enhancement ratio (ER) was calculated to evaluate the efficiency of the cubo-gel to improve the permeability in comparison with the drug solution. ER was calculated using the following equation: ${ }^{22,29}$

$$
E R=\frac{J_{24} \text { of the optimized formula }}{J_{24} \text { of the drug solution }}
$$

\section{In vivo DLX Bio-Distribution Study Administration and Sampling}

The animal experimental study protocol was approved by the research ethics committee for experimental and clinical studies at the Faculty of Pharmacy Cairo University [PI (2194)]. The study adheres to the principles of the ethical guidelines outlined by the International Council for Laboratory Animal Science (ICLAS). ${ }^{30,31}$ Swiss albino rats (No.: 46 and average weight: $100 \mathrm{gm}$ ) were randomly divided into four groups. The first and second groups administered the optimized in situ cubo-gel formulation and the drug solution, respectively, through the intranasal 
(IN) route. The third group was administered an intravenous (IV) formulation having the same composition of optimum cubo-gel except that the PF $127 \%$ was decreased to $10 \%$ to avoid gel formation after IV administration of the formulation. Finally, the fourth group was administered the drug solution intravenously. The following equation was applied to calculate the animal dose: ${ }^{32}$

$$
\text { Human dose }\left(\frac{m g}{k g}\right)=\text { Animal dose }\left(\frac{m g}{k g}\right) * \frac{\text { Animal } k m}{\text { Human } k m}
$$

The $\mathrm{K}_{\mathrm{m}}$ is the conversion factor and equals 37 and 6 for humans and rats, respectively.

The optimized formulation and drug solution used were both equivalents to $5 \mathrm{mg}$ DLX. Polyethene tube (inner diameter: $0.1 \mathrm{~mm}$ ), fitted to a Hamilton syringe was used for nasal administration. IV administration was done through the tail vein. At each time interval $(0.25,0.5$, $1,1.5,2,2.5,3,4,5,6,8,24,48$ and $72 \mathrm{~h}$ ) four rats, one from each group, were anaesthetized and sacrificed. From each rat, the brain was separated, and blood samples were collected. Clear plasma was separated by the centrifugation of the blood samples at $4000 \mathrm{rpm}$ for $15 \mathrm{~min}$. The plasma sample was kept in the freezer at $-80^{\circ} \mathrm{C}$ till analysis. Each separated brain was washed with normal saline (NS), weighed, and homogenized in PBS (pH 7.4) using a homogenizer (Heidolph DIAX 900, USA) to form 50\% homogenate. Samples were kept at $-80^{\circ} \mathrm{C}$ till analysis.

\section{Sample Preparation}

For the standard calibration curve preparation, defined volumes of DLX stock solution and $25 \mu \mathrm{L}$ of reboxetine solution $(5 \mathrm{mg} / \mathrm{mL})$, as an internal standard (IS); were added to $0.5 \mathrm{~mL}$ plasma to construct calibration standards with these concentrations: $0.1,0.2,1,5,20,40,60,80$, $100,150,200$ and $250 \mathrm{ng} / \mathrm{mL}$. For the sample preparation, $25 \mu \mathrm{L}$ of IS was added to a $0.5 \mathrm{~mL}$ sample (plasma or brain homogenate) and vortexed for $1 \mathrm{~min}$ in a $10 \mathrm{~mL}$ glass tube. Then, as protein precipitant, $4 \mathrm{~mL}$ of ethyl acetate was added to the previous sample, vortexed for 3 $\mathrm{min}$, and centrifuged for $10 \mathrm{~min}$ at $3000 \mathrm{rpm}$. The organic layer $(3 \mathrm{~mL})$ was separated into a new tube and dried under vacuum using a centrifugal vacuum concentrator at $45^{\circ} \mathrm{C}$ (Eppendorf 5301; Hamburg, Germany). Dry residues have been diluted in $500 \mu \mathrm{l}$ of the mobile phase, and $(20 \mu \mathrm{L})$ of this solution was injected into liquid chromatography with tandem mass spectrometry (LC-MS/MS) analysis. ${ }^{33}$

\section{LC-MS/MS}

Plasma and brain samples were analyzed using LC-MS /MS (Shimadzu, Japan) system fitted with a degasser (DGU-20A3) and solvent distribution unit (LC-20AD) and auto-sampler (SIL-20 A). A sensitive and validated LC-MS/MS system was established for the quantitative analysis of DLX using reboxetine as IS. The detection of MS/MS was done using AB Sciex API-3200 mass spectrometer (Foster City, CA, USA) fitted with a turbo ion spray interface at $550^{\circ} \mathrm{C}$ in positive ion mode. The ion spray voltage was adjusted to $5500 \mathrm{~V}$. For analysis, an aliquot of $25 \mu \mathrm{L}$ of the analyzed samples was injected on a $\mathrm{C} 18$ column, $100 \mathrm{~A}(50 \times 4.6 \mathrm{~mm})$ (Phenomenex, USA) with PS $5 \mu \mathrm{m}$. The mobile phase was isocratic and composed of acetonitrile and $0.5 \%$ formic acid in water at $80: 20(\mathrm{v} / \mathrm{v})$. The flow rate was adjusted to $1 \mathrm{~mL} / \mathrm{min}$. Ion detection was done using Multiple Reaction Monitoring (MRM) modes. In DLX, the transition was from $\mathrm{m} / \mathrm{z} 298.1$ precursor ion to $\mathrm{m} / \mathrm{z} 154.2$; in the case of reboxetine IS, it was from $\mathrm{m} / \mathrm{z}$ 314.2 precursor ion to $\mathrm{m} / \mathrm{z} 175.1$. The data have been analyzed using Version 1.4.2 of the Analyst Software (Applied Biosystems Inc., Foster City, USA). ${ }^{33}$

\section{Pharmacokinetics Parameters}

The pharmacokinetic profiles of DLX were described using the non-compartmental model after the IN administration of both the optimum formulation and drug solution to rats. The maximum concentration $\left(\mathrm{C}_{\max }\right)$ and the peak time (tmax) were obtained directly from the concentrationtime curve for plasma and brain. Other pharmacokinetic parameters such as elimination rate constant $\left(\mathrm{K}_{\mathrm{el}}\right)$, elimination half-life $\left(t_{1 / 2}\right)$, mean residence time (MRT), area under the curves from zero to the last time $\left(\mathrm{AUC}_{0-72}\right)$ and to infinity $\left(\mathrm{AUC}_{0 \text {-inf. }}\right)$ were calculated using Kinetica software version 5 (Thermo Fisher Scientific, MA, USA). Brain targeting efficiency was estimated by determining the brain targeting efficiency (BTE\%) and the drug transport percentage (DTP\%). BTE\% is the exposure of the brain to the drug after IN administration compared to that obtained by IV administration. It was calculated by the following equation: ${ }^{9,34}$

$$
B T E \%=\left(\frac{B_{I N} / P_{I N}}{B_{I V} / P_{I V}}\right) \times 100
$$

The $\mathrm{B}_{\mathrm{IN}}$ and $\mathrm{P}_{\mathrm{IN}}$ are the $\mathrm{AUC}_{0 \text {-inf. in brain homogenate (B) }}$ and plasma (P), respectively, after IN administration of optimized formulation and drug solution. On the other

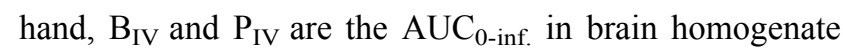


(B) and plasma (P), respectively, after IV administration. $\mathrm{BTE} \%$ of values range from 0 to $+\infty$. Efficient brain targeting after IN administration compared to IV is indicated by values above $100 \%{ }^{9}$

Furthermore, the DTP\% is the fraction of the drug transferred directly from the nose to the brain from the IN dose versus the total drug amount reaching the brain after IN delivery. It was calculated using the following equation: ${ }^{9,35}$

$$
\begin{gathered}
D T P \%=\left(\frac{B_{I N}-B_{X}}{B_{I N}}\right) \times 100 \\
B_{X}=\left(\frac{B_{I V}}{B_{I N}}\right) \times P_{I N}
\end{gathered}
$$

Positive DTP\% values up to $100 \%$ indicate that brain drug levels are to a great extent due to direct nose to brain transfer, whereas DTP\% equal to 0 (or even negative) indicates that the main brain entry pathway for the drug is through the systemic circulation after IV administration. ${ }^{9}$

\section{Stability Study}

A stability study was performed on DLX in situ cubo-gel to ensure its physical stability. An aliquot of $5 \mathrm{~mL}$ was taken from the formulation and stored in a tightly closed amber glass vial for 3 months at room temperature $\left(25^{\circ} \mathrm{C}\right)$ and refrigerator $\left(4^{\circ} \mathrm{C}\right)$. After the 3 months, the samples were evaluated by measuring its PS, PDI, ZP, and EE\%. All obtained data are the mean of three measurements $\pm \mathrm{SD}$.

\section{Statistical Analysis}

All data were expressed as mean \pm standard deviation (SD). The $3^{3} \mathrm{CCD}$ was generated and statistically analyzed using Design-Expert $^{\circledR}$ software (version 7, Stat-Ease Inc., MN, USA). Analysis of variance (ANOVA) was used to demonstrate the significance of each factor. The statistical analysis of the permeation study and the pharmacokinetic parameters was carried out using one way ANOVA test via SPSS 17.0 software (SPSS Inc., Chicago, USA). The differences between means were considered to be significant if the $p$-value was $<0.05$.

\section{Results and Discussion Characterization of the Prepared in situ Cubo-Gels PS, PDI, and ZP Analysis}

The PS of cubosomes had to be designed from 100 to $300 \mathrm{~nm}$ to achieve brain targeting. ${ }^{13}$ The PS of the prepared cubosomes ranged from $145.5 \mathrm{~nm}$ to $515.9 \mathrm{~nm}$, as shown in Table 2. This finding lies within the acceptable range for brain targeting. ${ }^{13}$ In a previous study, the PS of proniosomes prepared by Khatoon et al for brain targeting of DLX ranged from 166 to $842 \mathrm{~nm} .{ }^{19}$ The smallest size belonged to F4 prepared using 0.1 lipid ratio, 20\% w/v PF127 and 5\% w/v PF68. The linear model was the model of choice for the PS. The difference between adjusted $R^{2}$ and predicted $R^{2}$ must be less than 0.2 to indicate how the model can predict the response. PS model showed adjusted $\mathrm{R}^{2}(0.6613)$ and predicated $\mathrm{R}^{2}$ (0.538). The calculated equation for the PS analysis was:

$$
\mathrm{PS}=324.94+77.93 \mathrm{~A}-42.2 \mathrm{~B}+34.18 \mathrm{C}
$$

The effects of lipid ratio (A) and PF127\% (B) on the PS are shown in Figure 1A. ANOVA indicated that the lipid ratio and the PF127\% had a significant effect on PS $(p<0.05)$. Increasing the lipid ratio, decreasing PF127\%, and increasing PF68\% lead to larger PS. PF127 is a polymeric surfactant that acts as a stabilizer to the cubosomes. Increasing the concentration of PF127 stabilized the system sterically and made an efficient distribution of the crystalline structure of the cubosomes. ${ }^{36,37}$ Besides, increasing PF127 decreased the surface tension, which facilitated the formation of cubosomes with smaller PS. In a previous study, Abdelrahman, F.E. et al reported that increasing PF127 concentration led to a decrease in the PS of risperidone-prepared cubosomes. ${ }^{22}$ In another study, Khatoon et al reported that increasing Tween 80 concentration led to a reduction in PS of DLX proniosomes due to the decrease in surface tension. ${ }^{19} \mathrm{GMO}$ is an amphiphilic polar lipid. At concentration above CMC, it forms micelles. At higher concentrations, it forms cubosomes. ${ }^{13}$ Increasing the lipid ratio means increasing the GMO and increasing the PS of the formed cubosomes.

PDI is an indicator of homogeneity in PS distribution. $^{38}$ Lower values indicate monodispersity, while high values indicate polydispersity. PDI results ranged from 0.3 to 1 , as described in Table 2. F9 has the lowest PDI value of $0.222 \pm 0.02$.

The $\mathrm{ZP}$ is a stability indicator. It reflects the aggregation tendency of the nanoparticles. The greater the $\mathrm{ZP}$, the greater the force of repulsion that decreases particle aggregation. ${ }^{39}$ The ZP of the prepared cubosomes ranged from 1.6 to 10.6 mv, as shown in Table 2. The PF127 had a negative effect on the ZP because of its non-ionic nature. On the other hand, PF127 could act as a steric stabilizer that prevented the aggregation of the prepared cubosomes. ${ }^{12,40,41}$ 
Table 2 Average PS, GT, EE\%, Q6, PDI and ZP for the Prepared DLX in situ Cubo-Gels

\begin{tabular}{|c|c|c|c|c|c|c|}
\hline Formula & $\mathrm{PS} \pm \mathrm{SD}(\mathrm{nm})(\mathrm{YI})$ & $\mathrm{GT}\left({ }^{\circ} \mathrm{C}\right)(\mathrm{Y} 2)$ & EE\% \pm SD (\%) (Y3) & Q6 (\%) (Y4) & PDI \pm SD & $\mathrm{ZP} \pm \mathrm{SD}(\mathrm{mV})$ \\
\hline $\mathrm{FI}$ & $239 \pm 3$ & $80 \pm 2$ & $93.16 \pm 2.84$ & $34.87 \pm 2.8$ & $I \pm 0.1$ & $6.1 \pm 1.01$ \\
\hline F2 & $290 \pm 5$ & $80 \pm 2$ & $97.93 \pm 0.9$ & $26.9 \pm 3.65$ & $0.459 \pm 0.04$ & $2.33 \pm 0.58$ \\
\hline F3 & $276.1 \pm 6.24$ & $50 \pm 4.58$ & $97.3 \pm 1.28$ & $36.53 \pm 4.97$ & $0.582 \pm 0.03$ & $4 \pm 2.08$ \\
\hline $\mathrm{F} 4$ & $145.8 \pm 4.8$ & $34 \pm 2$ & $98.57 \pm 0.51$ & $26.43 \pm 2.25$ & $I \pm 0.1$ & $1.6 \pm 0.21$ \\
\hline F5 & $253 \pm 2.65$ & $36 \pm 2$ & $99.68 \pm 0.49$ & $64.5 \pm 4.13$ & $0.274 \pm 0.02$ & $1.6 \pm 0.08$ \\
\hline F6 & $384.9 \pm 4.56$ & $80 \pm 5$ & $96.9 \pm 0.07$ & $31.97 \pm 4.54$ & $0.508 \pm 0.07$ & $9.4 \pm 0.51$ \\
\hline F7 & $373.4 \pm 2.65$ & $50 \pm 3$ & $97.3 \pm 0.61$ & $29.83 \pm 4.38$ & $0.409 \pm 0.04$ & $7.8 \pm 1.35$ \\
\hline \multirow[t]{6}{*}{$\mathrm{F}^{\mathrm{a}}$} & $307.5 \pm 6.61$ & $45 \pm 1$ & $98.56 \pm 0.6$ & $38 \pm 2.36$ & $0.365 \pm 0.03$ & $10.6 \pm 1.8$ \\
\hline & $348.1 \pm 4.36$ & $45 \pm 2$ & $98.14 \pm 1$ & $39.5 \pm 2.21$ & $0.317 \pm 0.02$ & $8.33 \pm 1.15$ \\
\hline & $285 \pm 5$ & $45 \pm 1.63$ & $98.1 \pm 1.01$ & $37.07 \pm 5.33$ & $0.33 \pm 0.03$ & $9.87 \pm 1.32$ \\
\hline & $271.5 \pm 4.44$ & $45 \pm 3$ & $98.03 \pm 0.25$ & $38.7 \pm 1.67$ & $0.297 \pm 0.04$ & $8.2 \pm 3.79$ \\
\hline & $325.9 \pm 5.08$ & $45 \pm 3.61$ & $98.1 \pm 1$ & $36.2 \pm 4.55$ & $0.28 \mathrm{I} \pm 0.04$ & $9.7 \pm 3.79$ \\
\hline & $317.1 \pm 3.49$ & $45 \pm 2$ & $98.1 \pm 0.7$ & $39.8 \pm 2.71$ & $0.32 \pm 0.03$ & $8.9 \pm 2$ \\
\hline F9 & $331.4 \pm 4.61$ & $50 \pm 2$ & $99.03 \pm 0.75$ & $37.9 \pm 3.29$ & $0.222 \pm 0.02$ & $8.2 \pm 3.7$ \\
\hline FIO & $366.8 \pm 8.61$ & $34 \pm 2.65$ & $98.36 \pm 0.58$ & $35.53 \pm 1.76$ & $0.4865 \pm 0.01$ & $10 \pm 1.57$ \\
\hline FII & $377 \pm 2.65$ & $80 \pm 5$ & $98.13 \pm 0.52$ & $49.4 \pm 1.01$ & $0.43 I \pm 0.03$ & $10.5 \pm 1.53$ \\
\hline $\mathrm{F} 12$ & $515.9 \pm 5.29$ & $80 \pm 2.65$ & $99 \pm 0.52$ & $25.7 \pm 2.82$ & $0.675 \pm 0.02$ & $6.87 \pm 1.69$ \\
\hline $\mathrm{FI3}$ & $471.6 \pm 2.89$ & $52 \pm 2.65$ & $99 \pm 0.2$ & $31.87 \pm 3.31$ & $0.482 \pm 0.02$ & $9.1 \pm 2.85$ \\
\hline $\mathrm{FI} 4$ & $266 \pm 6.24$ & $32 \pm 1$ & $99.07 \pm 0.54$ & $20.3 \pm 2.14$ & $0.496 \pm 0.02$ & $6.67 \pm 3.06$ \\
\hline FI5 & $352.7 \pm 7.25$ & $37 \pm 2.65$ & $99.3 \pm 0.38$ & $30.63 \pm 1.46$ & $0.794 \pm 0.02$ & $7.6 \pm 1.25$ \\
\hline
\end{tabular}

Note: ${ }^{\mathrm{a}} \mathrm{F} 8$ represents the center point formulation and its five repetitions.

\section{Gelling Temperature (GT)}

The thermo-reversible in situ nasal gel is a free-flowing liquid at room temperature and transferred into a gel when inserted into the nasal cavity. ${ }^{15}$ The temperature at which the solution is transferred to gel is called the gelling temperature (GT). ${ }^{42}$ The GT of the designed formulations ranged from $32^{\circ} \mathrm{C}$ to $80^{\circ} \mathrm{C}$, as seen in Table 2 . The range $33^{\circ} \mathrm{C}-35^{\circ} \mathrm{C}$ is optimum for gelling inside the nasal cavity. ${ }^{43}$ The lowest GT $\left(32^{\circ} \mathrm{C} \pm 1\right)$ belonged to $\mathrm{F} 14$ which contains 20\% PF127 and 5\% PF68. The quadratic model was the most fitting model used for polynomial data analysis $(p<0.001)$. The predicated $R^{2}(0.9822)$ was in agreement with the adjusted $R^{2}(0.9550)$. The equation used to describe the GT was:

$\mathrm{GT}=46.55+0.1 \mathrm{~A}-22.7 \mathrm{~B}+0.7 \mathrm{C}-0.12 \mathrm{AB}+0.38 \mathrm{AC}$ $+0.88 \mathrm{BC}+2.14 \mathrm{~A}^{2}+8.14 \mathrm{~B}^{2}+1.14 \mathrm{C}^{2}$

ANOVA indicated that the GT was dependent only on PF $127 \%$ with $\mathrm{p}<0.001$. PF 127 had a negative effect on the GT; increasing PF $127 \%$ leads to a significant decrease in GT. The impact of PF127\% on the GT is shown in Figure 1B. Pluronics are linear triblock co-polymer consisted of polypropylene block (PPO) between two polyethylene oxide blocks (PEO). The amphiphilic characteristics depend on PPO and PEO length. Pluronic can be self-assembled into micelle with hydrophobic PPO core and hydrophilic PEO shell. By increasing concentration, gelation occurred at lower temperatures due to the packing of the micelles. PF127 and PF68 are the most frequent polymers used in the thermoreversible gel. PF127 forms gel at a lower temperature than PF68. Data analysis showed that the formulation containing 20\% PF127 formed a gel at a lower temperature. Adding PF68 to PF127 further decrease solgel transition temperature due to the hydrophilicity of PF68 which disrupts the hydration shell around PF127. ${ }^{24}$

\section{Entrapment Efficiency Percent (EE\%)}

As shown in Table 2, the obtained results revealed that EE $\%$ ranged from $93.1 \%$ to $99.7 \%$, indicating that the prepared cubosomes showed high drug entrapment. The twofactor interaction model was used with an acceptable difference between adjusted $R^{2}(0.96)$ and predicated $R^{2}$ (0.76). The equation describing the $\mathrm{EE} \%$ was:

$$
\mathrm{EE} \%=98.16+0.85 \mathrm{~A}+1.13 \mathrm{~B}+0.81 \mathrm{C}-0.66 \mathrm{AB}-0.68 \mathrm{AC}-
$$
$0.60 \mathrm{~B} \mathrm{C}$

Statistical ANOVA analysis indicated that all variables had a significant positive effect on EE\% $(p<0.001)$. Figure 1C and D represent the effect of lipid ratio, $\mathrm{PF} 127 \%$ and $\mathrm{PF} 68 \%$ on the $\mathrm{EE} \%$. GMO is an amphiphilic polar lipid. It can be self-assembled into bicontinuous cubic structures in water. ${ }^{12}$ The formed 
A

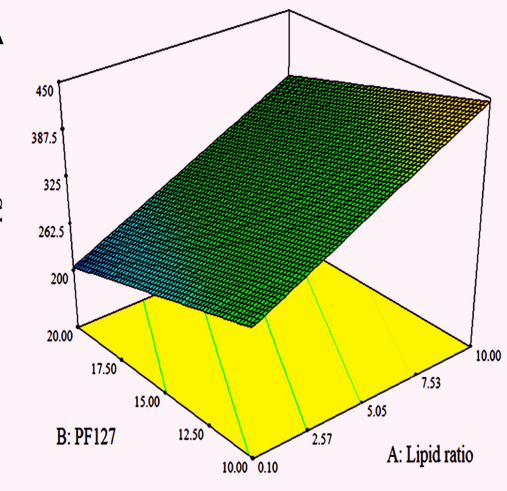

B

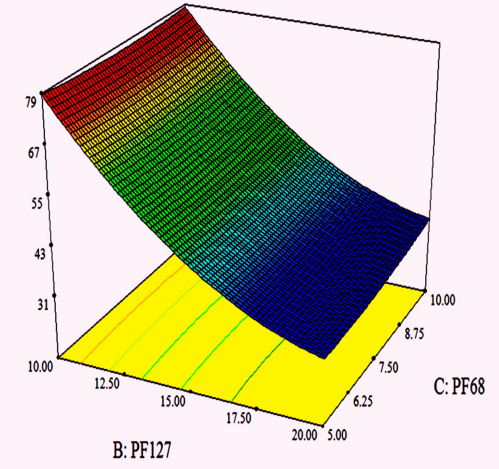

C

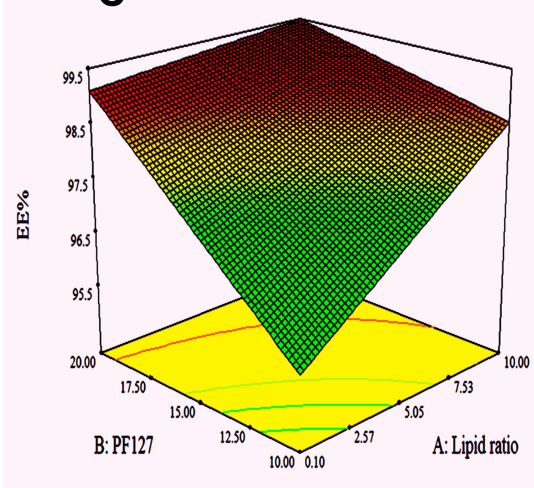

D

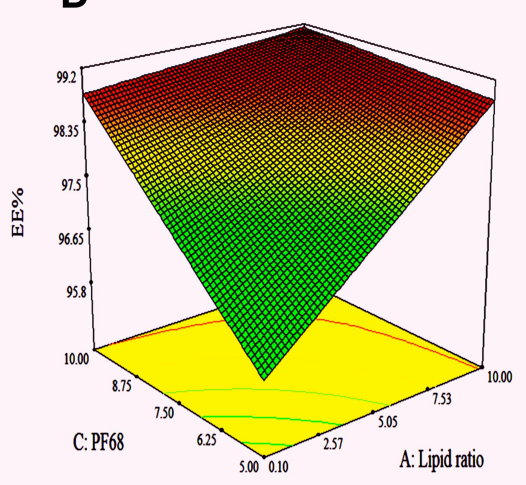

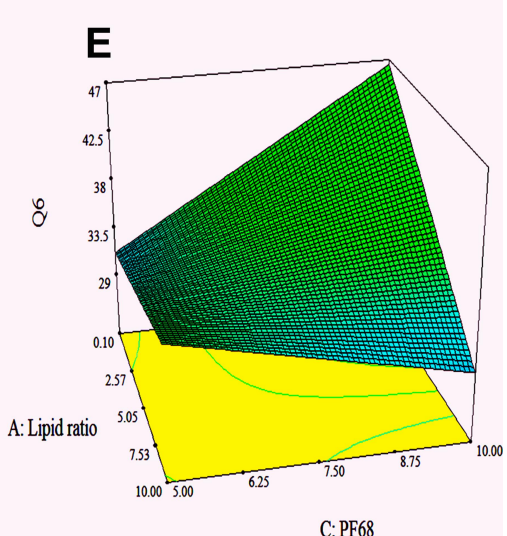

Figure I 3D surface plot for the main effects and interactions of lipid ratio, PFI27 percentage, and PF68 percentage on (A) PS (B) GT, (C and D) EE\%, and (E) Q6. The lipid ratio had a significant effect on PS, EE\%, and Q6; the PFI27\% had a significant impact on PS, GT, and EE\%; the PF68\% had a significant effect on EE\% and Q6.

Abbreviations: PFI27, Pluronic FI27; PF68, Pluronic F68; PS, particle size; GT, gelling temperature; EE\%, entrapment efficiency; Q6, percent released after 6 hours.

cubic structure had powerful encapsulation power for different drugs with different molecular weights and polarities. ${ }^{44}$ The second factor that affected the EE\% is PF $127 \%$. PF 127 is a water-soluble non-ionic triblock co-polymer with HLB $=22 .{ }^{45}$ PF127 has many functions in this design; it acts as a solubilizer to the drug, stabilizer to the cubosomes, and gelling agent. PF127 could increase the water solubility of the poorly soluble drugs and increase the drug entrapment in the water channels of the cubosomes. ${ }^{46}$ Besides, PF127 might stabilize the cubosomes entrapping the drug by forming a coat over them. The coat could retain an excess amount of DLX in such a way to increase its entrapment. ${ }^{37}$ The last factor that affected entrapment is PF68\%. Like the PF127, PF68 is a water-soluble copolymer with solubilizing efficiency, but less than PF127, so it could enhance the water solubility and the entrapment of a poorly soluble drug like DLX. ${ }^{46}$ In a previous study, it was stated that increasing Tween 80 concentration increased the EE\% of DLX in the prepared proniosomes due to the increased drug wettability. ${ }^{19}$ In another study, it was reported that increasing the Tween 80 concentration increased the $\mathrm{EE} \%$ of risperidone due to the formation of a coating layer over the prepared cubosomes, which retain additional drug amount. ${ }^{22}$

\section{In vitro DLX Release}

The DLX release from different cubo-gel formulations is represented in Figure 2, and the Q6 values are shown in Table 2. The formulations showed a slow drug release. The extent of DLX release after $24 \mathrm{~h}$ ranged from 42.77 \pm 7.01 to $101.38 \pm 1.7 \%$. In a previous study, it was reported that the extent of DLX release at $\mathrm{pH} 7.4$ after $24 \mathrm{~h}$ from the prepared proniosomal gel and mucoadhesive proniosomal gel was $30 \%$ and $24 \%$, respectively. ${ }^{19}$ This showed that our DLX cubo-gels could provide better-controlled release profiles and greater DLX extents compared to the formulations in the previous studies. Polynomial analysis, fitted with a 2 -factor interaction model, was used to illustrate the release data at Q6. There was an acceptable difference between adjusted $R^{2} \quad(0.908)$ and predicated $R^{2} \quad(0.729)$. 

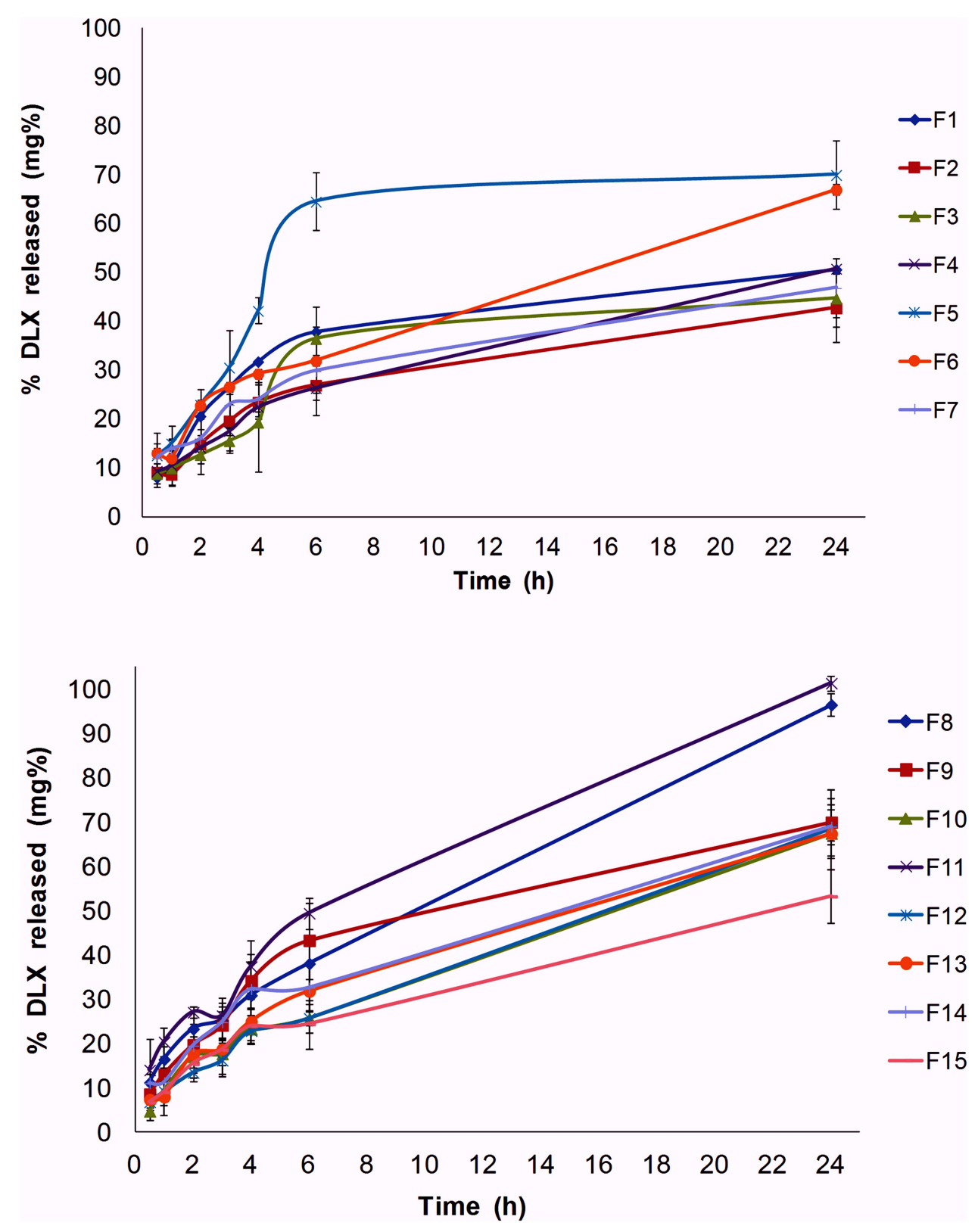

Figure 2 In vitro release profile of $\mathrm{DLX}$ in situ cubo-gels in PBS $(\mathrm{pH}=7.4)$ at $37^{\circ} \mathrm{C}$. Q6 ranged from $20.3 \pm 2.14$ to $64.5 \pm 4.13 \%$ and the release extent ranged from $42.77 \pm$ 7.01 to $101.38 \pm 1.7 \%$.

Abbreviations: DLX, duloxetine; PBS, phosphate buffer saline; Q6, percent released after 6 hours.

ANOVA showed that the lipid ratio and PF68\% were the factors significantly affecting the Q6 with $(\mathrm{p}<0.05)$. Figure 1E represents the effect of lipid ratio and PF68\% on the Q6. The equation describing the Q6 was:

$$
\mathrm{Q} 6=+35.63-3.24 \mathrm{~A}+0.74 \mathrm{~B}+2.38 \mathrm{C}-6.54 \mathrm{AB}-5.32 \mathrm{AC}
$$$$
+10.13 \mathrm{BC}
$$

The lipid ratio had a negative effect on the Q6 of the release. The GMO is the most common lipid used in cubosomes formulations; it might decrease the drug release by delaying drug partitioning from the oily medium to the aqueous medium. ${ }^{22}$ GTP is a hydrophobic triglyceride formed by the acylation of the three hydroxyl groups of the glycerol by palmitic acid. It could decrease the wettability of the formulation with the release medium and consequently reduced the drug release. ${ }^{47}$ On the other hand, PF68\% has a positive effect on the Q6; increasing the PF68\% lead to a significant increase in the drug release from the cubosomes. PF68 is amphiphilic co-polymer acting as a pore-forming agent and a release enhancer. ${ }^{48}$ 


\section{Characterization of the Optimized DLX in situ Cubo-Gel Formulation \\ PS, PDI, ZP, GT, EE\% and in vitro DLX Release}

The optimized formulation (composed of 3.76 lipid ratio, 20\% $\mathrm{w} / \mathrm{v}$ PF 127 , and 5\% w/v PF68) with desirability value $0.88 \mathrm{had}$ a PS of $265.13 \pm 9.85 \mathrm{~nm}$, PDI of $0.42 \pm 0.04$, and ZP of $2.79 \pm$ $0.44 \mathrm{mV}$. The GT was $32 \pm 0.05^{\circ} \mathrm{C}$. EE\% was $98.13 \pm 0.50 \%$, and the Q6 was 33\%. These findings were in agreement with the values predicted by the conducted statistical design.

\section{Gelling Time}

It is the time taken by the thermoresponsive formulations to form a gel (sol-to-gel transition) at a specific temperature. The cubo-gel transformed into gel after $10 \mathrm{sec}$ when kept at $33 \pm 0.5^{\circ} \mathrm{C}$.

\section{$\mathrm{pH}$ of the Formulation}

The $\mathrm{pH}$ of an intranasal product must be measured to avoid nasal mucosal irritation. The $\mathrm{pH}$ of an intranasal formulation must be from 5 to $6.5^{49}$ The cubo-gel $\mathrm{pH}$ was 6.3. This result is in agreement with that reported by Khatoon et al for DLX intranasal delivery. They reported that DLX proniosomal gel and mucoadhesive proniosomal gel had a $\mathrm{pH}$ of 6.44 and 5.67, respectively. ${ }^{19}$

\section{TEM Imaging}

It was observed that the cubosomes were not typical cubic in shape, as shown in Figure 3. In previous studies, Nasr et al and Abo El-Enin et al found that the drug-loaded cubosomes were nearly spherical with irregular polyangular shapes. ${ }^{10,37}$ This might be due to the GTP, which leads to a semi-cubic (semi-spherical) shape as it is reported in previous studies that GTP formed dense spherical nanoparticles. ${ }^{50,51}$ The scanned cubosomes showed numerous water channels in their structure as the GMO is self-assembled in water and form a liquid crystalline cubic phase consisted of bicontinuous lipid bilayers with networks of water channels. ${ }^{10}$ The cubosomes were well dispersed without aggregation; their surface was smooth and irregular. Their size was in agreement with that of the PS results. This PS was suitable for passing the $\mathrm{BBB}$ and achieving brain targeting.

\section{DSC}

DLX and optimized in situ cubo-gel DSC thermograms are shown in Figure 4. DLX thermogram displayed a high endothermic peak at $167.87^{\circ} \mathrm{C}$, indicating the crystalline state of the drug. ${ }^{3}$ However, the lyophilized cubosomes did not have a sharp peak but have two little peaks at $51.4^{\circ} \mathrm{C}$ and $65^{\circ} \mathrm{C}$. This could indicate that DLX, when formulated into the cubo-gel was encapsulated inside the cubosomes in an amorphous state or may have undergone an interaction.

\section{FTIR}

The FTIR spectra of DLX and lyophilized optimum in situ cubo-gel are shown in Figure 5. The FTIR
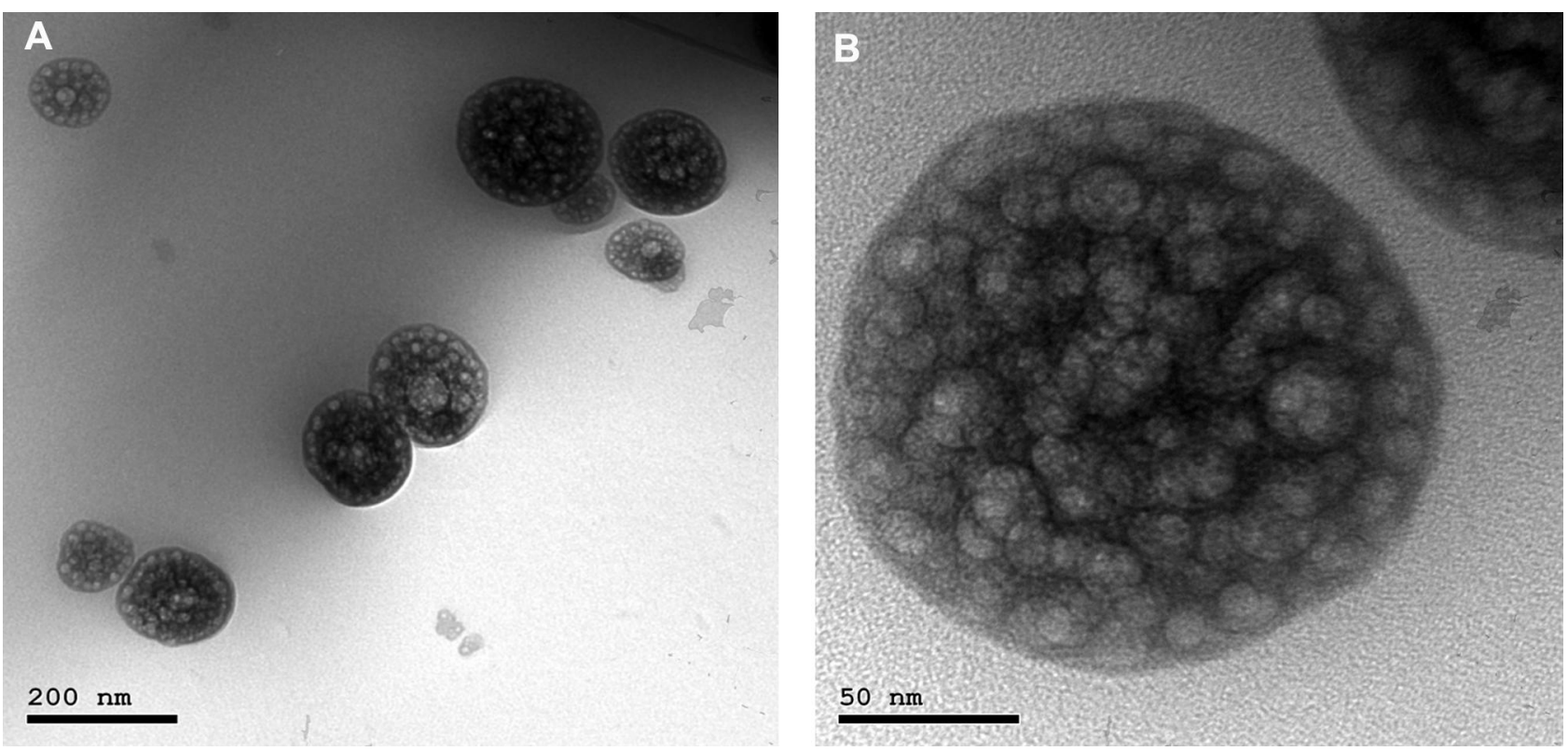

Figure 3 TEM images of (A) optimized DLX in situ cubo-gel formulation and (B) magnified single cubosome. The images show the cubosomes with numerous water channels in their structure.

Abbreviations: TEM, transmission electron microscope; DLX, duloxetine. 


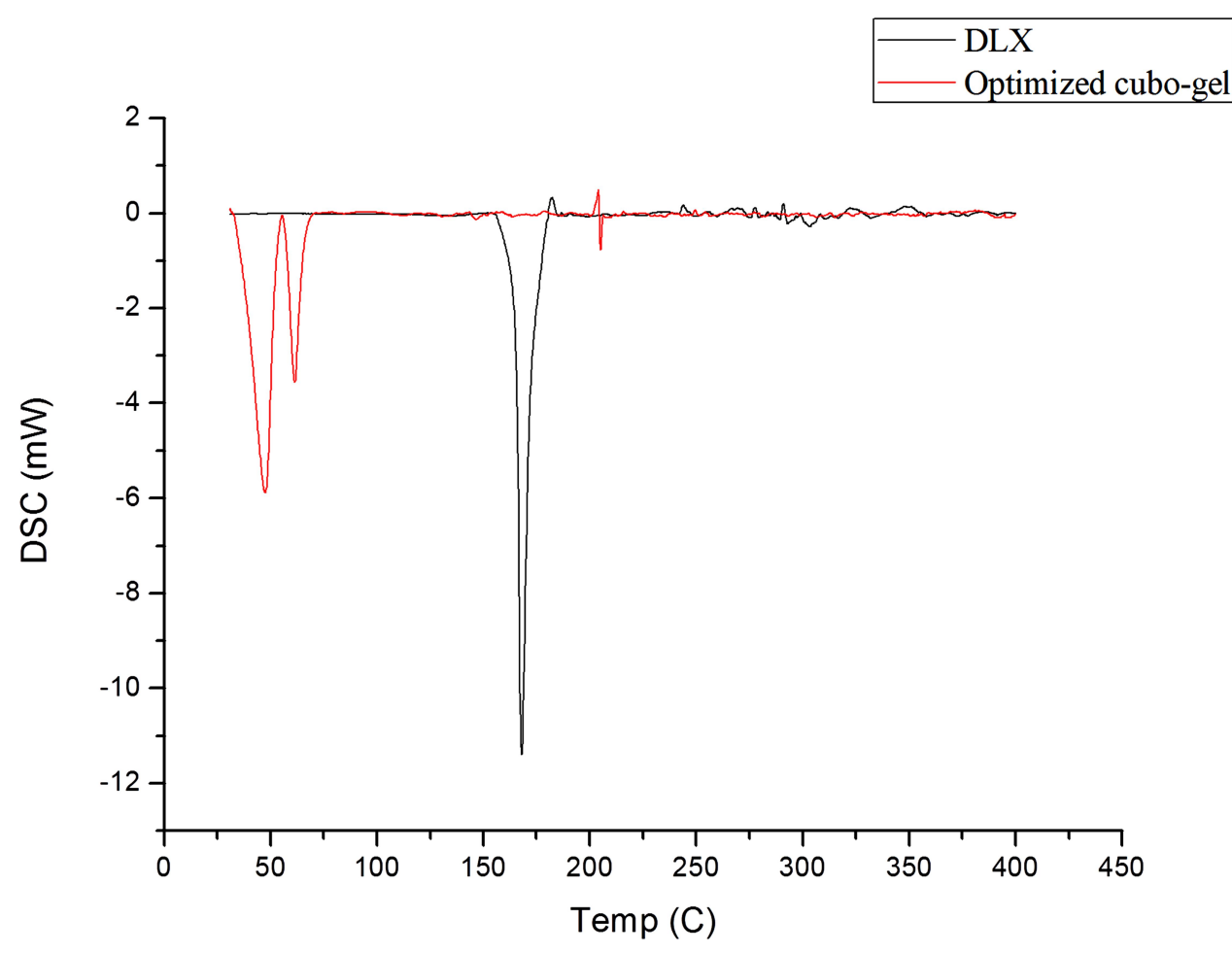

Figure 4 DSC thermograms of DLX showing an endothermic peak at $167.87^{\circ} \mathrm{C}$ and of optimum DLX in situ cubo-gel showing the disappearance of the DLX endothermic peak.

Abbreviations: DSC, differential scanning calorimetry; DLX, duloxetine.

spectra of GMO, ${ }^{52,53} \mathrm{GTP}^{50,51} \mathrm{PF} 127,{ }^{52,54}$ and $\mathrm{F} 68^{55}$ are obtained from previous studies found in the literature. The DLX spectrum showed distinct peaks at 1577.77 (aromatic alkenes), 1462.04 (thiophene ring), and 1234.44 (carbonyl group). ${ }^{56}$ The spectra of the lyophilized formulation showed a slight shift and a decrease in the peak strength of these groups. These findings were consistent with their DSC thermograms and might be due to the possible interaction between the GMO hydroxyl group and the carbonyl group in the drug. $^{57}$

\section{XRPD}

Figure 6 represents the XR diffractograms of DLX and the lyophilized optimized DLX loaded in situ cubo-gel. The DLX diffractogram showed distinct peaks at $18.2^{\circ}, 19.07^{\circ}$, $21.08^{\circ}, 23.5^{\circ}$, and $28.1^{\circ}(2 \theta)$ with relative intensity 45.32 , $60.06,100,50.43$, and 38.08 , respectively, reflecting its crystalline form. ${ }^{3}$ These peaks disappeared in the diffractogram of DLX loaded cubo-gel. This might indicate the molecular dispersion of the drug within the cubosomes, which leads to the loss of its crystalline nature and could indicate the complete encapsulation of the drug in the cubosomes.

\section{Cytotoxicity Assay}

The cytotoxicity test was conducted to ensure the safety of the cubo-gel components on the epithelial cells. As shown in Figure 7, the optimum plain in situ cubo-gel had a significantly higher $\mathrm{IC}_{50}(70.85 \mu \mathrm{g} / \mathrm{mL})$ compared to the drug solution and the drug-loaded in situ cubo-gel, which had almost similar $\mathrm{IC}_{50}$ values (21.66 and 20.77 $\mu \mathrm{g} / \mathrm{mL}$, respectively). The results showed that the formulation components were 3.27 times safer compared to the drug. Moreover, the formulation components had no synergistic effects on the drug cytotoxicity. Similar findings were observed by Ali-Boucetta et al and Desai et al who studied the safety of PF127 and lipid, respectively. ${ }^{58,59}$

\section{Ex vivo DLX Permeation Study}

The effect of the cubo-gel on DLX diffusion through the nasal membrane was assessed using the permeation study. This can help to predict the in vivo drug permeability. Figure 8 shows the permeation profiles of DLX loaded in situ cubo-gel compared to the DLX solution. DLX in situ cubo-gel and DLX solution showed drug flux $\left(\mathrm{J}_{24}\right)$ of 110.75 and $87.2 \mu \mathrm{g} / \mathrm{h} / \mathrm{cm}^{2}$, respectively. This indicated a significant increase in the permeation rate with $\mathrm{p}<0.001$. The enhancement ratio was 1.27 , indicating an increase in 

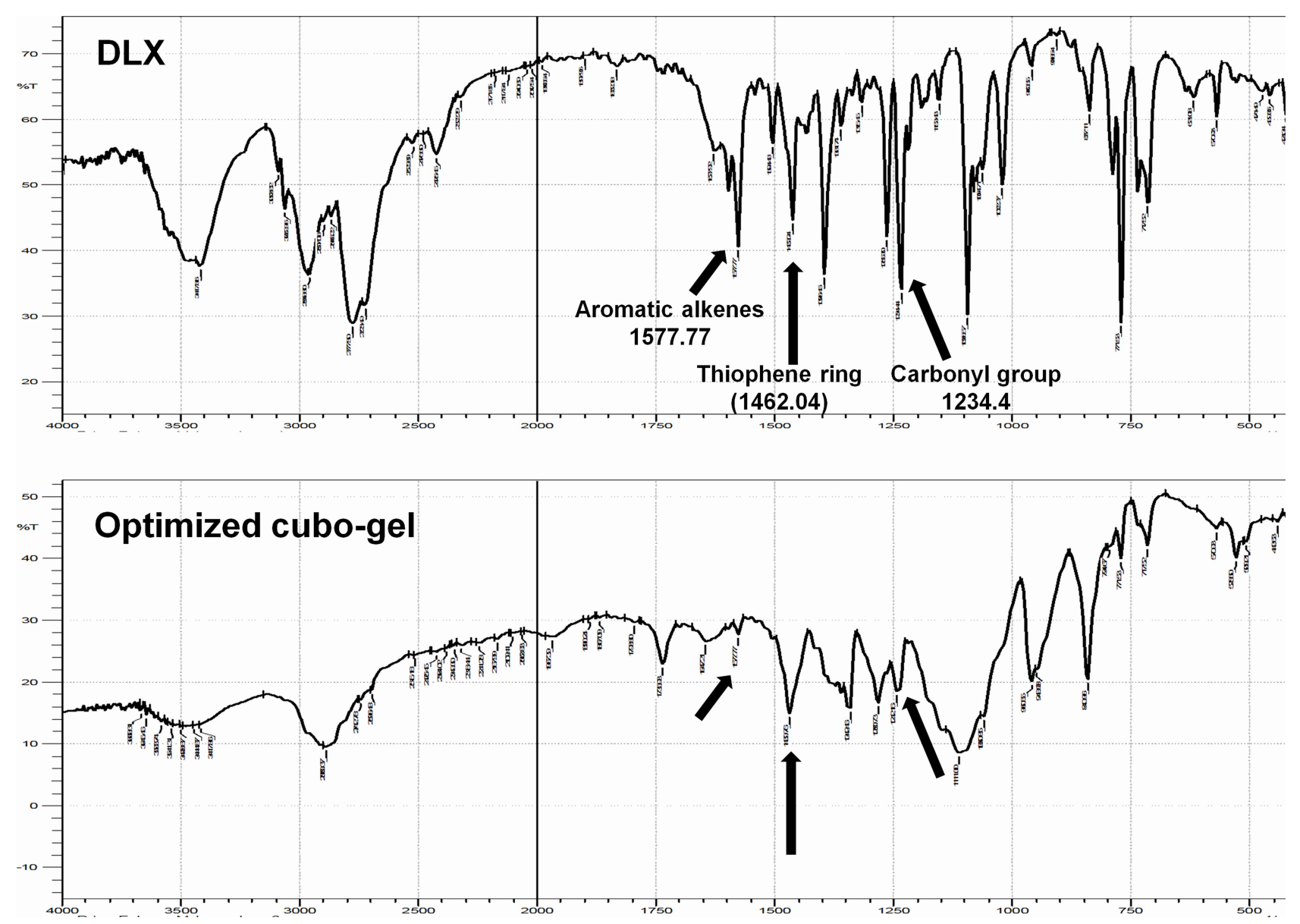

Figure 5 FTIR spectra of DLX and optimum DLX in situ cubo-gel showing shifted and reduced peaks at I577.77 (aromatic alkenes), I462.04 (thiophene ring), and I234.44 (carbonyl group).

Abbreviations: FTIR, Fourier-transform infrared spectroscopy; DLX, duloxetine.

the permeated drug amount per unit area through sheep nasal membrane from the cubo-gel compared to the DLX solution. A previous study reported that IN DLX proniosomal gel and mucoadhesive proniosomal gel showed a flux of 8.6 and $16.1 \mu \mathrm{g} / \mathrm{h} / \mathrm{cm}^{2}$, respectively. ${ }^{19}$ Our results showed that the DLX in situ cubo-gel had a flux of 110.75 $\mu \mathrm{g} / \mathrm{h} / \mathrm{cm}^{2}$. This confirms the superiority of our formulation in enhancing DLX permeation through nasal mucosa compared to previous studies.

Cubosomes' physical characters and the components involved in their structure might be the main reasons for the permeability improvement. Nano-sized drug delivery systems ranged from 1 to $1000 \mathrm{~nm}$ can enhance mucosal permeation and cellular internalization, and PS lower than $500 \mathrm{~nm}$ helps the nanoparticles to squeeze in the non-viscous aqueous pores found in the mucin network. ${ }^{9}$ In a previous study, Shilo et al found that the intracellular uptake of gold nanoparticles
(GNPs) was strongly dependent on GNPs PS. They stated that when the drug was encapsulated in the GNPs, the optimum size to cross the BBB and the brain cells was $70 \mathrm{~nm} .{ }^{60}$ In another study, Gao et al stated that the PS significantly influenced the delivery of methotrexate nanoparticles across the BBB. They noted that a significant difference in permeation across the BBB is found when the PS is below $100 \mathrm{~nm}$, but nanoparticles from 100 to $400 \mathrm{~nm}$ overcome the BBB non-significantly. ${ }^{61}$

Also, the PS influences the cellular uptake and internalization of the prepared cubosomes. Bourganis et al reported that nanoparticles with a diameter smaller than that of the olfactory axons (PS from 100 to $700 \mathrm{~nm}$ in humans) could be intracellularly transported to the brain via the olfactory neural pathway. ${ }^{62}$ Acosta concluded that nanoparticles with PS less than $500 \mathrm{~nm}$ had higher cellular uptake than nanoparticles with larger PS. ${ }^{63}$ 


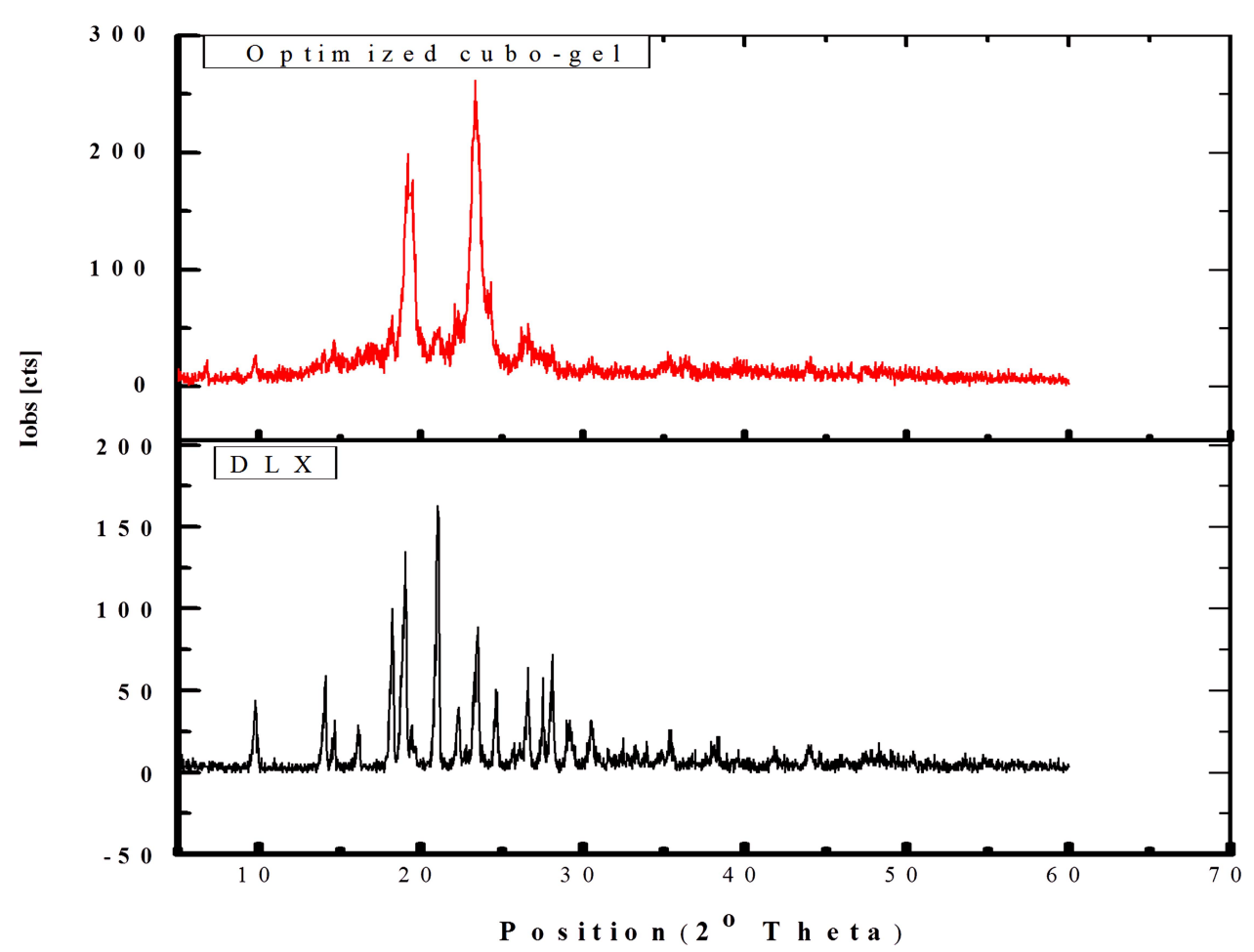

Figure 6 XRPD of DLX showing distinct peaks at $18.2^{\circ}, 19.07^{\circ}, 21.08^{\circ}, 23.5^{\circ}$, and $28.1^{\circ}$ and of optimum DLX in situ cubo-gel showing the disappearance of these peaks. Abbreviations: XRPD, X-ray powder diffraction; DLX, duloxetine.

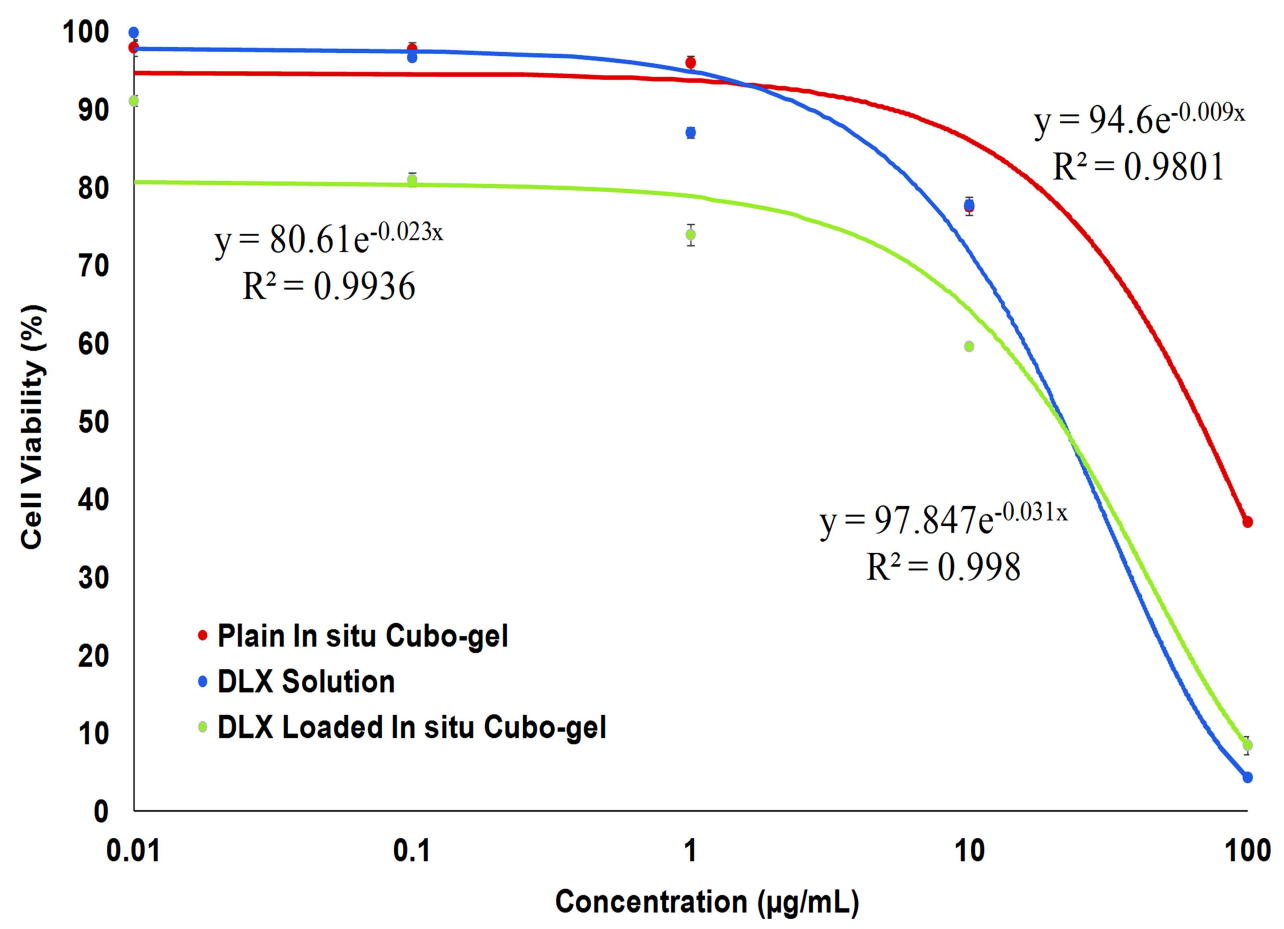

Figure 7 Cell viability of the optimized DLX in situ cubo-gel compared to plain in situ cubo-gel and DLX solution on oral epithelial cells showing that the plain in situ cubogel had a significantly higher $\mathrm{IC}_{50}(70.85 \mu \mathrm{g} / \mathrm{mL})$ compared to the $\mathrm{DLX}$ solution and the $\mathrm{DLX}$ in situ cubo-gel, which had almost similar $\mathrm{IC} \mathrm{C}_{50}$ values $(2 \mathrm{I} .66$ and $20.77 \mu \mathrm{g} / \mathrm{mL}$, respectively).

Abbreviations: DLX, duloxetine. 


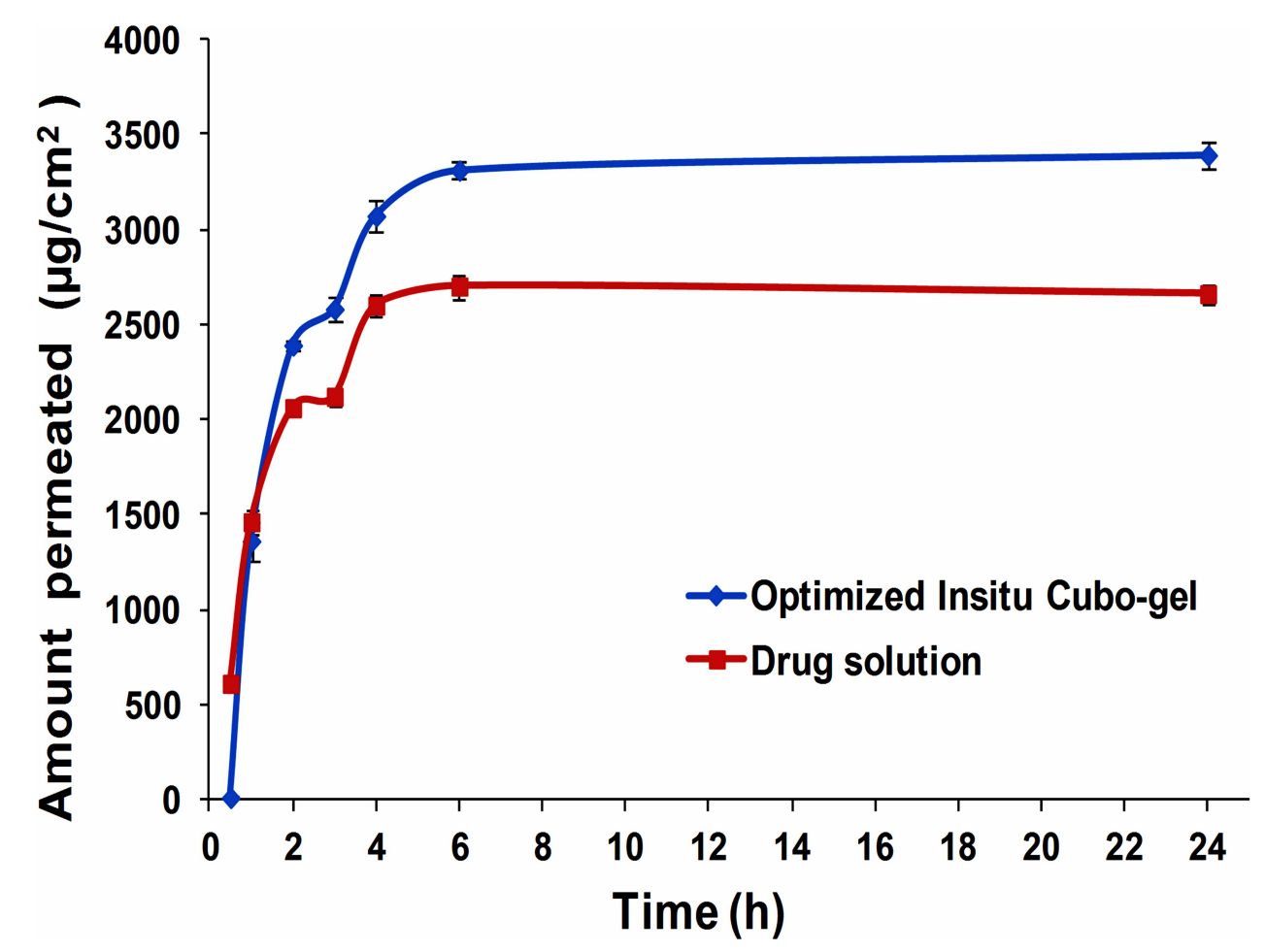

Figure 8 Cumulative amount of DLX permeated per unit area across the nasal sheep membrane via the optimized DLX in situ cubo-gel compared to the DLX solution showing increased permeation with 1.27 enhancement ratio.

Abbreviations: DLX, duloxetine.

Regarding the composition, PF127 and PF68 are copolymer surfactants with permeability enhancing ability, so improve mucosal penetration. GMO, a polar lipid, and the main component in cubosomes, could act as a permeability enhancer by promoting the intercellular lipid disorder by the interaction between its hydroxyl group and the anionic oxygen in the polar head of phospholipids membrane. ${ }^{64}$ These findings were in agreement with the results reported by Abdelrahman, F.E. et al who stated that the nanosize and the presence of Tween 80 in risperidone cubosomes lead to enhanced drug permeation across the nasal mucosa. ${ }^{22}$

\section{In vivo DLX Bio-Distribution Study}

The calibration curve of DLX showed a linear response from $0.1-250 \mathrm{ng} / \mathrm{mL}$, with a determination coefficient equal to 0.999 . The analytical method used in the biodistribution study was validated to ensure its accuracy and precision. The pharmacokinetics of DLX were studied in plasma and brain homogenate after intranasal (IN) and intravenous (IV) administration of both the formulation and the drug solution. The in vivo drug behavior in plasma and brain homogenate are shown in Figures 9 and 10. The pharmacokinetics parameters are shown in Table 3.
The pharmacokinetics results showed that the DLX level reached its peak concentration in the plasma and the brain after $0.15 \mathrm{~h}$ upon the IN administration of both the cubo-gel formulation and the drug solution. This short $t_{\max }$ might be attributed to the fast absorption through the IN route while the oral administration of DLX suffered from a $2 \mathrm{~h}$ lag time before absorption and the drug reached its $t_{\max }$ after $6 \mathrm{~h}^{65}$ The plasma $\mathrm{C}_{\max }$ of the IN cubo-gel was $215 \pm 7 \mathrm{ng} / \mathrm{mL}$, and the IN solution was $239.63 \pm 5.7$ $\mathrm{ng} / \mathrm{mL}$. In the brain tissue, the $\mathrm{C}_{\max }$ of the IN cubo-gel was $51.8 \pm 2.2 \mathrm{ng} / \mathrm{mL}$, while the $\mathrm{C}_{\max }$ of IN solution was 91.14 $\pm 4.15 \mathrm{ng} / \mathrm{mL}$. In a previous study, Alam et al prepared DLX nanostructured lipid carriers (NLC), which were administered as a circulating IN infusion in rats. The DLX NLC showed a drug concentration of $1869.247 \mu \mathrm{g} /$ $\mathrm{mL}$ in the plasma and $228.88 \mu \mathrm{g} / \mathrm{g}$ in the brain. ${ }^{18}$ The higher absorption and permeation of IN DLX NLC compared to our results might be attributed to many factors. First of all, the circulating DLX NLC IN infusion covered a larger area of the nasal mucosa for a more extended time in contrast to the DLX cubo-gel, which suffered from washing out by time. Secondly, the anesthesia of the rats to facilitate the administration of DLX NLC IN infusion could decrease the nasal mucociliary clearance and 


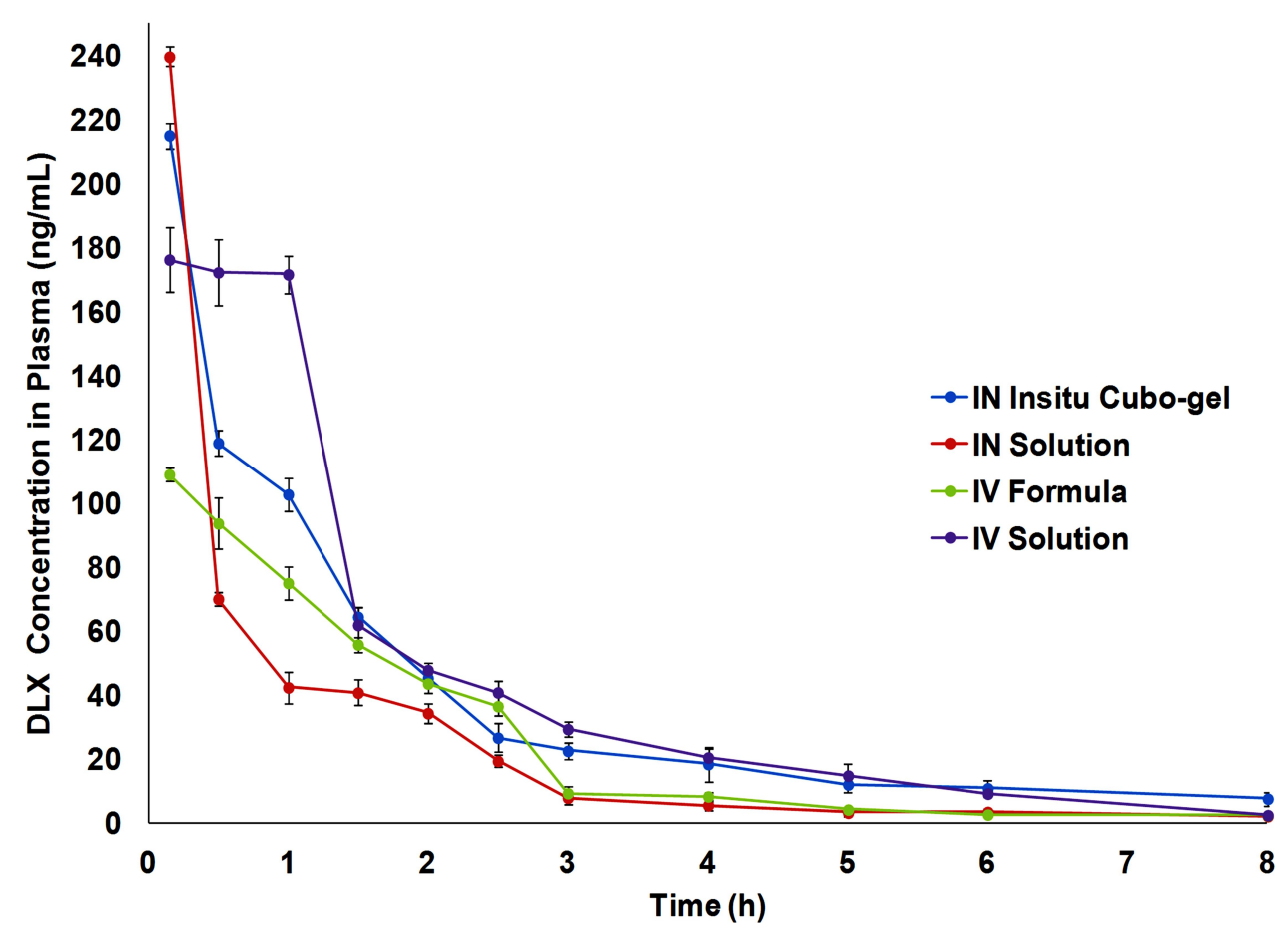

Figure 9 Mean plasma concentration-time profiles of IN DLX in situ cubo-gel in comparison to IN solution, IV formula, and IV solution after the administration in Swiss albino rats. The in situ cubo-gel showed higher $\mathrm{AUC}_{0 \text {-inf }}$ compared to the IN solution with relative bioavailability of $188.92 \%$.

Abbreviations: DLX, duloxetine; IN, intranasal; IV, intravenous; $A \cup C_{0 \text {-inf. }}$, area under the curves from zero to infinity.

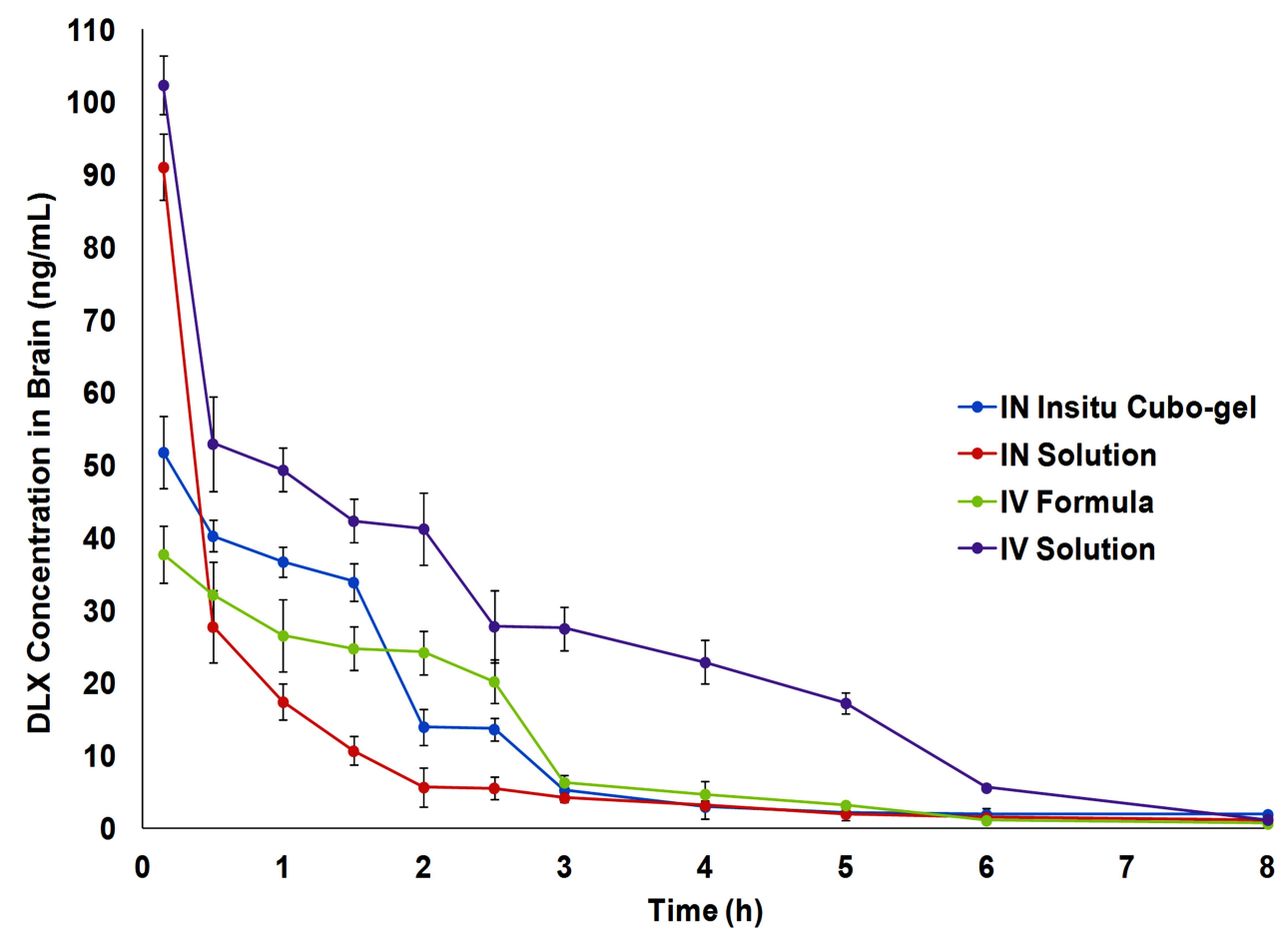

Figure 10 Mean brain homogenate concentration-time profiles of IN DLX in situ cubo-gel in comparison to IN solution, IV formula, and IV solution after the administration in Swiss albino rats. The in situ cubo-gel showed higher $\mathrm{AUC}_{0 \text {-inf }}$ compared to the IN solution with relative bioavailability of $196.13 \%$.

Abbreviations: DLX, duloxetine; IN, intranasal; IV, intravenous; $\mathrm{AUC}_{0 \text {-inf. }}$, area under the curves from zero to infinity. 


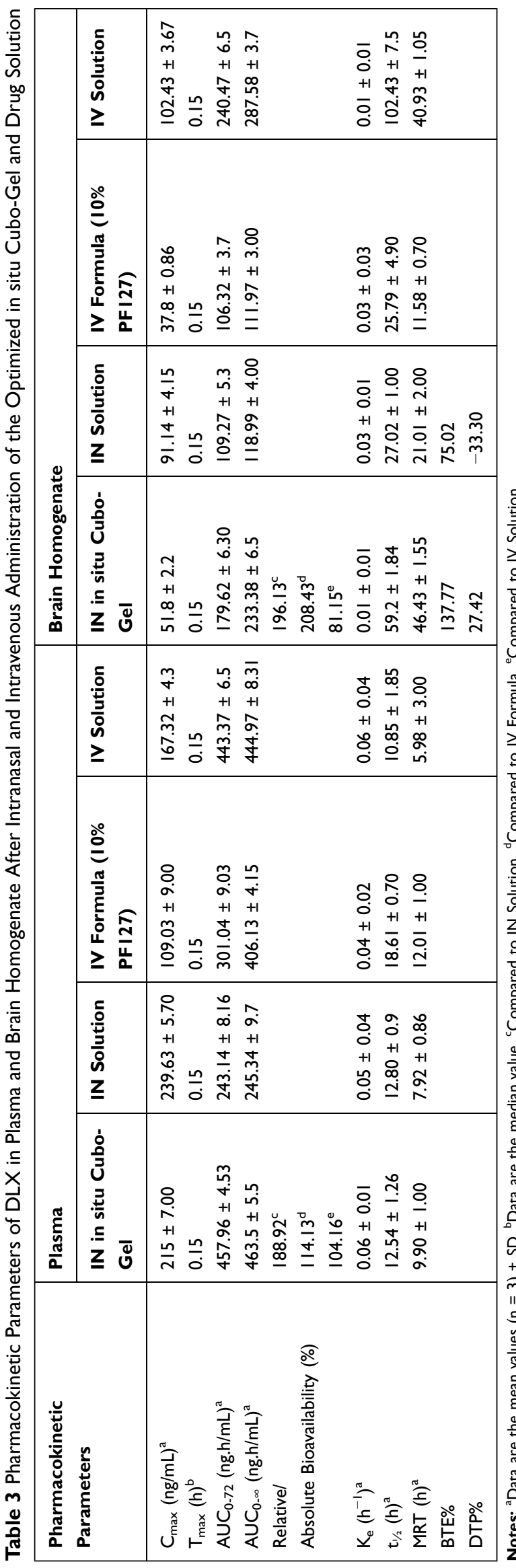

consequently increase the residence time and absorption of DLX NLC.

Although the IN solution had higher $\mathrm{C}_{\max }$ in both plasma and brain than the cubo-gel formulation, a significant enhancement in DLX bioavailability is achieved with the in situ cubo-gel as shown by the higher values of the $\mathrm{AUC}_{0-72}$ and $\mathrm{AUC}_{0-\infty}$ displayed in Table 3. The cubo-gel had a relative bioavailability of $188.92 \%$ and $196.13 \%$ in plasma and brain, respectively, compared to the IN solution. This might be due to the controlled release nature of the IN cubo-gel and its higher residence on the nasal mucosa, leading to lower $\mathrm{C}_{\max }$ at the beginning but higher bioavailability eventually.

The IN cubo-gel also showed a significantly higher bioavailability (208.43\%) in the brain than the IV formulation. This might be attributed to the direct transfer of the nasal formulation through the olfactory pathway to the brain and bypassing the BBB. On the other hand, the IN cubo-gel was bioequivalent to the IV solution in the plasma and the brain and bioequivalent to the IV formulation in the plasma. ${ }^{66}$

Additionally, the BTE\% of the IN cubo-gel (137.77\%) was significantly higher than that of the IN solution $(75.02 \%)$, indicating more efficient brain targeting by the IN cubo-gel. This might be attributed to the higher permeation of DLX from the cubo-gel either directly to the brain through the olfactory pathway or to some extent through the BBB after systemic absorption. These findings were further supported by DTP\%. The DTP\% of the IN cubo-gel was $10.5 \%$ but that of the IN solution was $13.7 \%$. The positive DTP\% in the IN cubo-gel indicated that the direct nose to brain pathway was responsible for the high DLX brain levels, while the negative DTP\% in the case of the IN solution indicated that DLX entered the brain preferentially via the systemic circulation after IV administration. ${ }^{9}$

Furthermore, the higher bioavailability and the superiority in brain targeting of the IN cubo-gel compared to the IN solution might be attributed to many other reasons. Firstly, the instilled formulation was transformed into a gel when inserted into the nasal cavity, leading to prolongation in the residence time of the formulation on the nasal mucosa. This overcame the rapid mucociliary washout, which is considered the most common problem with the IN route. ${ }^{49,67}$ Secondly, the lipophilic nature of the cubosomes allowed better vesicle penetration and more efficient drug absorption through the nasal mucosal membrane and the $\mathrm{BBB}$ for the systemically absorbed part of 
Table 4 Average PS, ZP, PDI and EE\% of Optimum DLX in situ Cubo-Gel After Storage for 3 Months at Room Temperature and Refrigerator

\begin{tabular}{|l|l|l|l|l|}
\hline & $\begin{array}{l}\text { PS } \pm \text { SD } \\
(\mathbf{n m})\end{array}$ & $\begin{array}{l}\text { ZP } \pm \text { SD } \\
(\mathbf{m V})\end{array}$ & PDI \pm SD & $\begin{array}{l}\text { EE\% } \pm \text { SD } \\
(\%)\end{array}$ \\
\hline $\begin{array}{l}\text { Initial } \\
\text { Room } \\
\begin{array}{l}\text { Temperature } \\
\text { Refrigerator }\end{array}\end{array}$ & $265.13 \pm 9.85$ & $2.79 \pm 0.44$ & $0.41 \pm 0.05$ & $98.13 \pm 0.50$ \\
& $272.17 \pm 8.72$ & $2.85 \pm 0.02$ & $0.37 \pm 0.01$ & $97.20 \pm 1.01$ \\
\hline
\end{tabular}

the formulation. Thirdly, Bourganis et al reported a correlation between the surface charge and the DTP\%. In particular, it can be observed that as the ZP approaches zero, higher DTP\% values can be achieved. ${ }^{62}$ Finally, according to the $\mathrm{BTE} \%$ and $\mathrm{DTP} \%$, the larger proportion of the drug reached the brain directly through the olfactory pathway, which is considered a unique access gate to the brain as it is devoid of the BBB protection. 9, 68,69

\section{Stability Study}

The results in Table 4 showed that there were minimal changes in cubosomes physical characters. In the case of room temperature, there is slight decrease in PS and EE\%. In contrast, in the refrigerator $\left(4-8^{\circ} \mathrm{C}\right)$, it was found that there is a slight increase in PS and a minimal decrease in $\mathrm{EE} \%$. ZP was also minimally changed at both room temperature and refrigerator. The little changes in PS, ZP, and $\mathrm{EE} \%$ were statistically insignificant $(\mathrm{P}<0.05$, paired $t$-test). The presence of a high concentration of PF127 and PF68 allowed system stability due to their amphiphilic nature. ${ }^{45}$

\section{Conclusion}

Thermoreversible DLX in situ cubo-gel was successfully prepared with suitable GT, high EE\%, small and uniform PS, and controlled drug release. Statistical analysis of the $3^{3} \mathrm{CCD}$ showed that the lipid ratio had a significant effect on $\mathrm{PS}, \mathrm{EE} \%$, and Q6; the PF127\% had a substantial effect on PS, GT, and EE\%; the PF68\% had a significant impact on EE\% and Q6. Optimum DLX in situ cubo-gel with the desirability of 0.88 was prepared and evaluated. It had PS of $265.13 \pm$ $9.85 \mathrm{~nm}, \mathrm{GT}$ of $32 \pm 0.05^{\circ} \mathrm{C}, \mathrm{EE} \%$ of $98.13 \pm 0.50 \%$, and Q6 of 33\%. The cytotoxicity study revealed that the formulation components were 3.27 times safer compared to the drug. The ex vivo permeation study showed a 1.27 enhancement ratio in the flux, indicating an increased drug permeation from the DLX in situ cubo- gel. The in vivo bio-distribution study showed that the IN in situ cubo-gel is much better than the IN solution in bioavailability enhancement and brain targeting. The IN in situ cubo-gel showed a 1.96 fold increase in brain bioavailability compared to the IN solution. The BTE\% and $\mathrm{DTP} \%$ of the in situ cubo-gel were $137.77 \%$ and $10.5 \%$, respectively, indicating efficient brain targeting after IN administration. The GMO and Pluronics incorporated in the cubo-gel formulation have powerful solubilizing and penetration enhancing effects improving the DLX encapsulation in the cubosomes and their absorption through the nasal cavity directly to the brain. Based on these studies, the IN DLX in situ cubo-gel could be considered as an innovative nano-carrier delivery system for brain targeting of DLX to maximize its effect.

\section{Abbreviations}

DLX, duloxetine; BCS, biopharmaceutics classification system; CMC, critical micelle concentration; PEO, polyethylene oxide; PPO, polypropylene; CCD, central composite design; GMO, glycerol monooleate; GTP glycerol tripalmitate; PF127, Pluronic F127; PF68, Pluronic F68; PS, particle size; PDI, polydispersity index; ZP, zeta potential; GT, gelling temperature; EE\%, entrapment efficiency; Q6, percent released after 6 hours; SD, standard deviation; PBS, phosphate buffer saline; TEM, transmission electron microscope; DSC, differential scanning calorimetry; FTIR, Fourier-transform infrared spectroscopy; XRPD, X-ray powder diffraction; $\mathrm{J}_{24}$, drug flux at 24 hours; ER, enhancement ratio; IN, intranasal; IV, intravenous; IS, internal standard; LC-MS/MS, liquid chromatography with tandem mass spectrometry; $\mathrm{C}_{\max }$, maximum concentration; $\mathrm{t}_{\max }$, time for the peak; $\mathrm{K}_{\mathrm{el}}$, elimination rate constant; $t_{1 / 2}$, elimination half-life; MRT, mean residence time; $\mathrm{AUC}_{0-72}$, area under the curves from zero to the last time; $\mathrm{AUC}_{0 \text {-inf., }}$ area under the curves from zero to infinity; $\mathrm{BTE} \%$, brain targeting efficiency; DTP\%, drug transport percentage.

\section{Acknowledgment}

The author would like to acknowledge Mr. Dellvin Roshon Williams, Lecturer of English in the Department of General Education at Gulf Medical University, UAE, for proofreading and editing the manuscript.

\section{Disclosure}

The authors report no conflicts of interest in this work. 


\section{References}

1. Saminathan J, Sankar ASK, Anandakumar K, Vetrichelvan T. Simple UV spectrophotometric method for the determination of fluvastatin sodium in bulk and pharmaceutical formulations. E J Chem. 2009;6 (4):1233-1239. doi:10.1155/2009/530206

2. Bymaster FP, et al. Comparative affinity of duloxetine and venlafaxine for serotonin and norepinephrine transporters in vitro and in vivo, human serotonin receptor subtypes, and other neuronal receptors. Neuropsychopharmacology. 2001;25(6):871-880. doi:10.1016/S089 3-133X(01)00298-6

3. Pandya P, Pandey NK, Singh SK, Kumar M. Formulation and characterization of ternary complex of poorly soluble duloxetine hydrochloride. J Appl Pharm Sci. 2015;5(6):088-096. doi:10.7324/ JAPS.2015.50615

4. Ganesh M, Ubaidulla U, Hemalatha P, Peng MM, Jang HT. Development of duloxetine hydrochloride loaded mesoporous silica nanoparticles: characterizations and in vitro evaluation. AAPS PharmSciTech. 2015;16(4):944-951. doi:10.1208/s12249-014-0273-x

5. Patel K, Padhye S, Nagarsenker M. Duloxetine HCl lipid nanoparticles: preparation, characterization, and dosage form design. AAPS PharmSciTech. 2012;13(1):125-133. doi:10.1208/s12249-011-9727-6

6. Alam MI, Baboota S, Ahuja A, Ali M, Ali J, Sahni JK. Intranasal administration of nanostructured lipid carriers containing CNS acting drug: pharmacodynamic studies and estimation in blood and brain. $J$ Psychiatr Res. 2012;46(9):1133-1138. doi:10.1016/j.jpsychires.2012.05.014

7. Mathison S, Nagilla R, Kompella UB. Nasal route for direct delivery of solutes to the central nervous system: fact or fiction? J Drug Target. 1998;5(6):415-441. doi:10.3109/10611869808997870

8. Wang X, Chi N, Tang X. Preparation of estradiol chitosan nanoparticles for improving nasal absorption and brain targeting. Eur J Pharm Biopharm. 2008;70(3):735-740. doi:10.1016/j.ejpb.2008. 07.005

9. Sonvico F, Clementino A, Buttini F, et al. Surface-modified nanocarriers for nose-to-brain delivery: from bioadhesion to targeting. Pharmaceutics. 2018;10(1):34. doi:10.3390/pharmaceutics10010034

10. Nasr M, Ghorab MK, Abdelazem A. In vitro and in vivo evaluation of cubosomes containing 5-fluorouracil for liver targeting. Acta Pharm Sin B. 2015;5(1):79-88. doi:10.1016/j.apsb.2014.12.001

11. Kulkarni CV, Vishwapathi VK, Quarshie A, et al. Self-assembled lipid cubic phase and cubosomes for the delivery of aspirin as a model drug. Langmuir. 2017;33(38):9907-9915. doi:10.1021/acs. langmuir. $7 \mathrm{~b} 02486$

12. Gaballa S, El Garhy O, Abdelkader H. Cubosomes: composition, preparation, and drug delivery applications. J Adv Biomed Pharm Sci. 2019;3(1):1-9. doi:10.21608/jabps.2019.16887.1057

13. Ahirrao M, Shrotriya S. In vitro and in vivo evaluation of cubosomal in situ nasal gel containing resveratrol for brain targeting. Drug Dev Ind Pharm. 2017;43(10):1686-1693. doi:10.1080/03639045.2017.13 38721

14. Salama HA, Mahmoud AA, Kamel AO, Abdel Hady M, Awad GAS. Phospholipid based colloidal poloxamer-nanocubic vesicles for brain targeting via the nasal route. Colloids and Surfaces B: Biointerfaces. 2012;100:146-154. doi:10.1016/j.colsurfb.2012.05.010

15. Jagdale S, Shewale N, Kuchekar BS. Optimization of thermoreversible in situ nasal gel of timolol maleate. Scientifica. 2016;6401267. doi: $10.1155 / 2016 / 6401267$

16. Kumar R, Kumar S, Sinha VR. Evaluation and optimization of water-in-oil microemulsion using ternary phase diagram and central composite design. J Dispers Sci Technol. 2016;37(2):166-172. doi:10.1080/01932691.2015.1038351

17. Fares AR, Elmeshad AN, Kassem MAA. Enhancement of dissolution and oral bioavailability of lacidipine via pluronic P123/F127 mixed polymeric micelles: formulation, optimization using central composite design and in vivo bioavailability study. Drug Deliv. 2018;25 (1):132-142. doi:10.1080/10717544.2017.1419512
18. Alam MI, Baboota S, Ahuja A, Ali M, Ali J, Sahni JK. Intranasal infusion of nanostructured lipid carriers (NLC) containing CNS acting drug and estimation in brain and blood. Drug Deliv. 2013;20 (6):247-251. doi:10.3109/10717544.2013.822945

19. Khatoon M, Sohail MF, Shahnaz G, et al. Development and evaluation of optimized thiolated chitosan proniosomal gel containing duloxetine for intranasal delivery. AAPS PharmSciTech. 2019;20 (7):288. doi:10.1208/s12249-019-1484-y

20. Soga O, Van Nostrum CF, Fens M, et al. Thermosensitive and biodegradable polymeric micelles for paclitaxel delivery. $J$ Control Release. 2005;103(2):341-353. doi:10.1016/j.jconrel.2004.12.009

21. Zaki NM, Awad GA, Mortada ND, Abd ElHady SS. Enhanced bioavailability of metoclopramide $\mathrm{HCl}$ by intranasal administration of a mucoadhesive in situ gel with modulated rheological and mucociliary transport properties. Eur J Pharm Sci. 2007;32(4-5):296-307. doi:10.1016/j.ejps.2007.08.006

22. Abdelrahman FE, Elsayed I, Gad MK, Badr A, Mohamed MI. Investigating the cubosomal ability for transnasal brain targeting: in vitro optimization, ex vivo permeation and in vivo biodistribution. Int J Pharm. 2015;490(1-2):281-291. doi:10.1016/j. ijpharm.2015.05.064

23. Yang SC, Lu LF, Cai Y, Zhu JB, Liang BW, Yang CZ. Body distribution in mice of intravenously injected camptothecin solid lipid nanoparticles and targeting effect on brain. J Control Release. 1999;59(3):299-307. doi:10.1016/S0168-3659(99)00007-3

24. Rarokar NR, Saoji SD, Raut NA, Taksande JB, Khedekar PB, Dave VS. Nanostructured cubosomes in a thermoresponsive depot system: an alternative approach for the controlled delivery of docetaxel. AAPS PharmSciTech. 2016;17(2):436-445. doi:10.1208/ s12249-015-0369-y

25. Sherafudeen SP, Vasantha PV. Development and evaluation of in situ nasal gel formulations of loratadine. Res Pharm Sci. 2015;10 (6):466-476.

26. Elzahhar PA. Expanding the anticancer potential of 1,2,3-triazoles via simultaneously targeting Cyclooxygenase-2, 15-lipoxygenase and tumor-associated carbonic anhydrases. Eur J Med Chem. 2020;200:112439. doi:10.1016/j.ejmech.2020.112439

27. Fathy U, Azzam MA, Mahdy F, El-Maghraby S, Allam RM. Synthesis and in vitro anticancer activity of some novel tetrahydroquinoline derivatives bearing pyrazol and hydrazide moiety. $J$ Heterocycl Chem. 2020;57(5):2108-2120. doi:10.1002/jhet.3 930

28. El Zaafarany GM, Awad GAS, Holayel SM, Mortada ND. Role of edge activators and surface charge in developing ultradeformable vesicles with enhanced skin delivery. Int J Pharm. 2010;397(1-2):164-172. doi:10.1016/j.ijpharm.2010.06.034

29. Al-Mahallawi AM, Khowessah OM, Shoukri RA. Nanotransfersomal ciprofloxacin loaded vesicles for non-invasive trans-tympanic ototopical delivery: in-vitro optimization, ex-vivo permeation studies, and in-vivo assessment. Int J Pharm. 2014;472 (1-2):304-314. doi:10.1016/j.ijpharm.2014.06.041

30. Osborne N, Avey MT, Anestidou L, Ritskes-Hoitinga M, Griffin G. Improving animal research reporting standards. EMBO Reports. 2018;19(5):5. doi:10.15252/embr.201846069

31. ICLAS Ethical Guideline for Researchers. http://iclas.org/guidelinesfor-researchers.

32. Reagan-Shaw S, Nihal M, Ahmad N. Dose translation from animal to human studies revisited. FASEB J. 2008;22(3):659-661. doi:10.1096/ fj.07-95741sf

33. El Sharawy AM, Shukr MH, Elshafeey AH. Formulation and optimization of duloxetine hydrochloride buccal films: in vitro and in vivo evaluation. Drug Deliv. 2017;24(1):1762-1769. doi:10.1080/ 10717544.2017.1402216

34. Vyas T, Shahiwala A, Marathe S, Misra A. Intranasal drug delivery for brain targeting. Curr Drug Deliv. 2005;2(2):165-175. doi:10.21 $74 / 1567201053586047$ 
35. Kozlovskaya L, Abou-Kaoud M, Stepensky D. Quantitative analysis of drug delivery to the brain via nasal route. $J$ Control Release. 2014;189:133-140. doi:10.1016/j.jconrel.2014.06.053

36. Younes NF, Abdel-Halim SA, Elassasy AI. Corneal targeted Sertaconazole nitrate loaded cubosomes: preparation, statistical optimization, in vitro characterization, ex vivo permeation and in vivo studies. Int J Pharm. 2018;553(1-2):386-397. doi:10.1016/j.ijpharm.2018.10.057

37. El-enin HA, Al-shanbari AH. Nanostructured liquid crystalline formulation as a remarkable new drug delivery system of anti-epileptic drugs for treating children patients. Saudi Pharm J. 2018;26 (6):790-800. doi:10.1016/j.jsps.2018.04.004

38. Clayton KN, Salameh JW, Wereley ST, Kinzer-Ursem TL. Physical characterization of nanoparticle size and surface modification using particle scattering diffusometry. Biomicrofluidics. 2016;10(5):05410 7. doi:10.1063/1.4962992

39. Williams PM. Zeta Potential. Encyclopedia Membranes. 2016; 2063-2064. doi:10.1007/978-3-662-44324-8 612

40. Koo OMY. Pharmaceutical Excipients: Properties, Functionality, and Applications in Research and Industry; 2016. doi:10.1002/ 9781118992432

41. Chong JYT, Mulet X, Waddington LJ, Boyd BJ, Drummond CJ. Steric stabilisation of self-assembled cubic lyotropic liquid crystalline nanoparticles: high throughput evaluation of triblock polyethylene oxide-polypropylene oxide-polyethylene oxide copolymers. Soft Matter. 2011;7(10):4768-4777. doi:10.1039/c1sm05181d

42. Miller SC, Donovan MD. Effect of poloxamer 407 gel on the miotic activity of pilocarpine nitrate in rabbits. Int J Pharm. 1982;12(2-3):147-152. doi:10.1016/0378-5173(82)90114-4

43. Foxman EF, Storer JA, Fitzgerald ME, et al. Temperature-dependent innate defense against the common cold virus limits viral replication at warm temperature in mouse airway cells. Proc Natl Acad Sci U S A. 2015;112(3):827-832. doi:10.1073/pnas.1411030112

44. Shah JC. Cubic phase gels as drug delivery systems. Adv Drug Deliv Rev. 2001;47(2-3):229-250. doi:10.1016/S0169-409X(01)00108-9

45. Giuliano E, Paolino D, Fresta M, Cosco D. Mucosal applications of poloxamer 407-based hydrogels: an overview. Pharmaceutics. 2018;10(3):1-26. doi:10.3390/pharmaceutics 10030159

46. Ban E, Park M, Jeong S, et al. Poloxamer-based thermoreversible gel for topical delivery of emodin: influence of P407 and P188 on solubility of emodin and its application in cellular activity screening. Molecules. 2017;22(2):246. doi:10.3390/molecules22020 246

47. Yehia SA, Elshafeey AH, Elsayed I. Biodegradable donepezil lipospheres for depot injection: optimization and in-vivo evaluation. J Pharm Pharmacol. 2012;64(10):1425-1437. doi:10.1111/j.20427158.2012.01530.x

48. Mei L, Zhang Y, Zheng Y, et al. A novel docetaxel-loaded poly ( $\varepsilon-$ caprolactone)/Pluronic F68 nanoparticle overcoming multidrug resistance for breast cancer treatment. Nanoscale Res Lett. 2009;4 (12):1530-1539. doi:10.1007/s11671-009-9431-6

49. Bhatt M. An overview: formulation and product development of nasal spray. World J Pharm Res. 2017;404-413. doi:10.20959/ wjpr20176-8557

50. Patil J, Rajput R, Nemade R, Naik J. Preparation and characterization of artemether loaded solid lipid nanoparticles: a 32 factorial design approach. Mater Technol. 2020;35(11-12):719-726. doi:10.1080/ 10667857.2018.1475142

51. Yousry C, Fahmy RH, Essam T, El-laithy HM, Elkheshen SA. Nanoparticles as tool for enhanced ophthalmic delivery of vancomycin: a multidistrict-based microbiological study, solid lipid nanoparticles formulation and evaluation. Drug Dev Ind Pharm. 2016;42 (11):1752-1762. doi:10.3109/03639045.2016.1171335

52. Patil S, Kadam C, Pokharkar V. QbD based approach for optimization of Tenofovir disoproxil fumarate loaded liquid crystal precursor with improved permeability. $J$ Adv Res. 2017;8(6):607-616. doi:10.1016/j. jare.2017.07.005
53. Ekambaram P, Abdul Hasan Sathali A. Abdul Hasan Sathali A. Formulation and evaluation of solid lipid nanoparticles of ramipril. J Young Pharm. 2011;3(3):216-220. doi:10.4103/0975-14 83.83765

54. Karolewicz B, Gajda M, Górniak A, Owczarek A, Mucha MI. Pluronic F127 as a suitable carrier for preparing the imatinib base solid dispersions and its potential in development of a modified release dosage forms. $J$ Therm Anal Calorim. 2017;130(1):383-390. doi:10.1007/s10973-017-6139-1

55. Patil S, Ujalambkar V, Rathore A, Rojatkar S, Pokharkar V. Galangin loaded galactosylated pluronic F68 polymeric micelles for liver targeting. Biomed Pharmacother. 2019;112:108691. doi:10.1016/j. biopha.2019.108691

56. Singh A, Bali A. Formulation and characterization of transdermal patches for controlled delivery of duloxetine hydrochloride. $J$ Anal Sci Technol. 2016;7(1):25. doi:10.1186/s40543-016-0105-6

57. Hashem F, Nasr M, Youssif YM. Formulation and characterization of cubosomes containing REB for improvement of oral absorption of the drug in human volunteers. $J$ Adv Pharm Res. 2018;2(2):95-103. doi:10.21608/aprh.2018.5828

58. Ali-Boucetta H, Al-Jamal KT, Müller KH, et al. Cellular uptake and cytotoxic impact of chemically functionalized and polymer-coated carbon nanotubes. Small. 2011;7(22):3230-3238. doi:10.1002/smll. 201101004

59. Desai HH, Bu P, Shah AV, Cheng X, Serajuddin ATM. Evaluation of cytotoxicity of self-emulsifying formulations containing long-chain lipids using Caco-2 cell model: superior safety profile compared to medium-chain lipids. $J$ Pharm Sci. 2020;109(5):1752-1764. doi:10.1016/j.xphs.2020.01.031

60. Shilo M, Sharon A, Baranes K, Motiei M, Lellouche J-PM, Popovtzer R. The effect of nanoparticle size on the probability to cross the blood-brain barrier: an in-vitro endothelial cell model. J Nanobiotechnology. 2015;13(1):19. doi:10.1186/s12951-015-00 $75-7$

61. Gao K, Jiang X. Influence of particle size on transport of methotrexate across blood brain barrier by polysorbate 80 -coated polybutylcyanoacrylate nanoparticles. Int $J$ Pharm. 2006;310(1-2):213-219. doi:10.1016/j.ijpharm.2005.11.040

62. Bourganis V, Kammona O, Alexopoulos A, Kiparissides C. Recent advances in carrier mediated nose-to-brain delivery of pharmaceutics. Eur J Pharm Biopharm. 2018;128:337-362. doi:10.1016/j.ejpb.20 18.05.009

63. Acosta E. Bioavailability of nanoparticles in nutrient and nutraceutical delivery. Curr Opin Colloid Interface Sci. 2009;14(1):3-15. doi:10.1016/j.cocis.2008.01.002

64. Pereira GR, Collett JH, Garcia SB, Thomazini JA, Bentley MVLB. Glycerol monooleate/solvents systems for progesterone transdermal delivery: in vitro permeation and microscopic studies. Revista Brasileira De Ciências Farmacêuticas. 2002;38(1):55-62. doi:10.15 90/S1516-93322002000100005

65. Bymaster FP, Beedle EE, Findlay J, et al. Duloxetine (Cymbalta ${ }^{\mathrm{TM}}$ ), a dual inhibitor of serotonin and norepinephrine reuptake. Bioorganic Med Chem Lett. 2003;13(24):4477-4480. doi:10.1016/j.bmcl.2003.08.079

66. Chow S-C. Bioavailability and bioequivalence in drug development. Wiley Interdiscip Rev Comput Stat. 2014;6(4):304-312. doi:10.1002/wics.1310

67. Swamy NGN, Abbas Z. Mucoadhesive in situ gels as nasal drug delivery systems: an overview. Asian J Pharm Sci. 2012;7 (3):168-180.

68. Mahajan HS, Mahajan MS, Nerkar PP, Agrawal A. Nanoemulsionbased intranasal drug delivery system of saquinavir mesylate for brain targeting. Drug Deliv. 2014;21(2):148-154. doi:10.3109/1071 7544.2013.838014

69. Natarajan J, Baskaran M, Humtsoe LC, Vadivelan R, Justin A. Enhanced brain targeting efficacy of Olanzapine through solid lipid nanoparticles. Artificial Cells, Nanomedicine, and Biotechnology. 2017;45(2):364-371. doi:10.3109/21691401.2016.1160402 


\section{Publish your work in this journal}

The International Journal of Nanomedicine is an international, peerreviewed journal focusing on the application of nanotechnology in diagnostics, therapeutics, and drug delivery systems throughout the biomedical field. This journal is indexed on PubMed Central, MedLine, CAS, SciSearch ${ }^{\mathbb{B}}$, Current Contents ${ }^{\mathbb{B}} /$ Clinical Medicine,
Journal Citation Reports/Science Edition, EMBase, Scopus and the Elsevier Bibliographic databases. The manuscript management system is completely online and includes a very quick and fair peer-review system, which is all easy to use. Visit http://www.dovepress.com/ testimonials.php to read real quotes from published authors.

Submit your manuscript here: https://www.dovepress.com/international-journal-of-nanomedicine-journal 